\title{
3D numerical modelling of turbidity currents
}

Anastasios N. Georgoulas ${ }^{1}$, Panagiotis B. Angelidis ${ }^{1}$, Theologos G. Panagiotidis ${ }^{2}$, Nikolaos E.

\author{
Kotsovinos ${ }^{1}$, \\ ${ }^{1}$ Laboratory of Hydraulics and Hydraulic Structures \\ Democritus University of Thrace, Department of Civil Engineering, V. Sofias 12 \\ GR-67 100 Xanthi, Greece \\ ${ }^{2}$ Laboratory of Fluid Mechanics \\ Institute of Technology, Mechanical Department, Agios Loukas \\ GR-654 04 Kavala, Greece \\ Tel: +302541079321, FAX: +302541079604 \\ ageorg@civil.duth.gr
}

\begin{abstract}
During floods, the density of river water usually increases due to a subsequent increase in the concentration of the suspended sediment that the river carries, causing the river to plunge underneath the free surface of a receiving water basin and form a turbidity current that continues to flow along the bottom. The study and understanding of such complex phenomena is of great importance, as they constitute one of the major mechanisms for suspended sediment transport from rivers into oceans, lakes or reservoirs. Unlike most of the previous numerical investigations on turbidity currents, in this paper, a 3D numerical model that simulates the dynamics and flow structure of turbidity currents, through a multiphase flow approach is proposed, using the commercial CFD code FLUENT. A series of numerical simulations that reproduce particular published laboratory flows are presented. The detailed qualitative and quantitative comparison of numerical with laboratory results indicates that apart from the global flow structure, the proposed numerical approach efficiently predicts various important aspects of turbidity current flows, such as the effect of suspended sediment mixture composition in the temporal and spatial evolution of the simulated currents, the interaction of turbidity currents with loose sediment bottom layers and the formation of internal hydraulic jumps. Furthermore, various extreme cases among the numerical runs considered are further analyzed, in order to identify the importance of various controlling flow parameters.
\end{abstract}

Keywords: Turbidity currents, hyper-pycnal flows, CFD numerical modelling, suspended sediment transport. 


\section{Introduction}

In nature, there is a large class of flows that are generated and driven by the density difference between two or even more fluids. These flows are known as gravity or density currents. Despite the fact that the density difference between two fluids usually arises due to differences in temperature or salinity, it can also arise due to the presence of suspended solid particles. These particulate currents, in the case of sediment laden water that enters a water basin, are classified according to the density difference with the ambient fluid into three major categories: a) hypopycnal currents, when the density of the sediment laden water is lower than that of the receiving water basin, b) homopycnal currents, when the density of the sediment laden water is almost equal to that of the receiving water basin, and c) hyperpycnal currents when their density is much greater than that of the receiving water body [1]. In the case of floods, the suspended sediment concentration of river water rises to a great extent. Hence, the river plunges to the bottom of the receiving basin and forms a hyperpycnal plume which is also known as turbidity current. Such flows are usually formed at river mouths in oceans, lakes or reservoirs, and can travel remarkable distances transferring, eroding and depositing large amounts of suspended sediments [1].

Turbidity currents are very difficult to be observed and studied in the field. This is due to their rare and unexpected occurrence nature, as they are usually formed during floods. Field investigations are usually limited to the study of the deposits originating from such currents. The anatomy of deposits originating from turbidity currents can be studied on a large scale, in order to identify the various depositional elements such as lobes, levees and submarine channels [2]. Furthermore, considerable research on the morphology of turbiditic systems and general deep-marine depositions is being increasingly done with the use of 3D seismic sections $[3,4]$.

On the other hand, scaled laboratory experiments constitute an alternative and widely used method for simulating and studying the dynamics of turbidity currents. Many researchers have been focused in the study of the flow dynamics, depositional and erosional characteristics of laboratory turbidity currents, using scaled experimental models [5-8]. Advances in experimental technology in the last decades have increased the existing knowledge from macroscopic and qualitative descriptions of turbidity current behaviour and deposits, to detailed, quantitative results relating to the actual flow characteristics, such as the velocity, concentration as well as the turbulence structure of such flows [9-12].

Mathematical and numerical models when properly designed and tested against field 
or laboratory data, can provide significant knowledge for turbidity current dynamics as well as erosional and depositional characteristics. Up to present, there are various numerical investigations dealing with turbidity current dynamics and flow characteristics, providing valuable results regarding these complex phenomena. The characteristics of a gravity-current head have been studied by Hartel et al. [13], using 3D Direct Numerical Simulations (DNS) of flow fronts in the lock-exchange configuration. Kassem and Imran [14] present a 2D numerical approach for investigating the transformation of a plunging river flow into a turbidity current. In the work of Heimsund et al. [15] a computational, 3D, fluid-dynamics model for sediment transport, erosion and deposition by turbidity currents has been constructed using the CFD software Flow-3D. Another 3D numerical model using the CFX-4 code was developed, in order to simulate turbidity currents in Lake Lugano (Switzerland), in the work of Lavelli et al. [16]. Necker et al. [17] presented 2D and 3D Direct Numerical Simulations of particle-driven gravity currents, placing special emphasis on the sedimentation of particles, and the influence of particle settling on the flow dynamics. Cantero et al. [18] present two and three-dimensional CFD simulations of a discontinuous density current, using a stabilized equal-order finite element method. A comparative study on the convergence of CFD commercial codes, when simulating dense underflows is presented by Bombardelli et al. [19]. Two codes are used for the proposed simulations: the first one is a comprehensive finiteelement platform, whereas the other one is a commercial code. The lateral development of density-driven flow in a subaqueous channel is studied using a 3D numerical model, in the work of Imran et al. [20]. The conditions under which turbidity currents may become selfsustaining through particle entrainment are investigated in the work of Blanchette et al. [21], using 2D Direct Numerical Simulations of resuspending gravity currents. A numerical model of turbidity currents with a deforming bottom boundary, that predicts the vertical structure of the flow velocity and concentration as well as the change in the bed level, due to erosion and deposition of suspended sediment, is developed in the work of Huang et al. [22]. Lockexchange gravity current flows, produced by the instantaneous release of a heavy fluid, are investigated by means of 2D Large-Eddy Simulation (LES) in the work of Ooi et al. [23]. A numerical simulation of turbidity current using the $\overline{v^{2}}-f$ turbulence model is carried out in the work of Mehdizadeh et al. [24]. Cantero et al. [25], perform 2D Direct Numerical Simulations in order to investigate the effect of particle inertia on the dynamics of particulate gravity currents. They introduce an Eulerian-Eulerian formulation for gravity currents driven by inertial particles. 3D Direct Numerical Simulations of planar gravity currents have been 
conducted with the objective of identifying, visualizing and describing turbulent structures and their influence on flow dynamics, in the work of Cantero et al. [26]. The investigation of the effect of initial aspect ratio on the flow characteristics of suspension gravity currents as well as the diffusion of the turbidity under the presence of a turbidity fence is carried out in the work of Singh [27], using 3D Large Eddy Simulations.

In this paper, the use of Computational Fluid Dynamics (CFD) methods offered by the commercial software FLUENT, in order to simulate the 3D dynamics and flow behaviour of turbidity currents, is proposed. The numerical model used is based on a multiphase modification of the Reynolds Averaged Navier-Stokes equations (RANS). For turbulence closure the Renormalization-group (RNG) $k-\varepsilon$ model is applied, which is an enhanced version of the widely used standard $k-\varepsilon$ model. Unlike most of the previous CFD investigations that treat the turbidity current flow as a quasi single-phase approach, solving one set of continuity and momentum equations for the ambient fluid and treating the transport of sediment particles through an advection-diffusion equation for sediment concentration, the present multiphase numerical approach assumes that the sediment-laden turbidity current flow consists of separate solid and fluid phases which are treated as interpenetrating continua. The major advantage of this different multiphase approach is that a separate velocity field is calculated for each phase (water and sediment classes), since the laws for the conservation of mass and momentum are modified accordingly in order to be satisfied by each phase individually. In the first part of the present paper, after the description of the numerical model, the numerical model results are compared in detail with the results of previously published laboratory experiments. For this purpose two different series of laboratory experiments on turbidity currents are reproduced numerically, including both simple lock-gate flume experiments [10] as well as more complex experiments on high-density turbidity currents that interact with loose sediment beds [11]. The main aim is to justify the ability of the numerical model in capturing various important aspects of turbidity currents, such as their flow structure, the effect of suspended sediment mixture composition in their temporal and spatial evolution, their interaction with loose sediment bottom layers and the formation of internal hydraulic jumps. In the second part of the present paper, further processing and analysis of the numerical results is conducted aiming to identify the effect and importance of various controlling parameters, in the flow structure as well as the spatial and temporal evolution of turbidity currents. For this purpose detailed comparison of various results between extreme cases among the numerical runs considered is performed, both for the lock-gate and the loose bed cases. 


\section{Model Description}

\subsection{General Information}

Turbidity current flows can be characterized as multiphase flow systems, since they consist of a primary fluid phase (water) and secondary granular phases (suspended sediment classes) dispersed into the primary phase. Therefore, turbidity currents can be modeled through the application of suitable multiphase numerical models. Since, the particulate loading of turbidity currents may vary from small to considerably large values an EulerianEulerian multiphase numerical approach is considered to be more appropriate, as it can handle a wider range of particle volume fractions than an Eulerian-Lagrangian approach (maximum particles volume fraction of 10-12\%). FLUENT provides various multiphase models that are based in the Eulerian-Eulerian approach. The Eulerian model that has been chosen for the simulations of the present paper may require more computational effort, but it can handle a wider range of particulate loading values and is more accurate than the other available multiphase models in FLUENT. In this multiphase model, the different phases are treated mathematically as interpenetrating continua and therefore the concept of phasic volume fraction is introduced, where the volume fraction of each phase is assumed to be a continuous function of space and time. The sum of the volume fractions of the various phases is equal to unity. An accordingly modified set of momentum and continuity equations for each phase is solved. Pressure and inter-phase exchange coefficients are used in order to achieve coupling for these equations. The coupling of granular (fluid-solid) flows is handled differently than in the case of non-granular (fluid-fluid) flows. For granular flows, the properties are obtained from application of the kinetic theory. The type of phases involved, also defines the momentum exchange between the various phases [28].

The motion of the suspended sediment particles within a turbidity current as well as the motion generated in the ambient fluid are of highly turbulent nature. In order to account for the effect of turbulence in the numerical simulations of the present investigation, the instantaneous governing equations are not applied directly but they are ensemble-averaged, converting turbulent fluctuations into Reynolds stresses, which represent the effects of turbulence. This averaging procedure for the numerical simulation of turbulent flows is known as RANS (Reynolds-averaged Navier-Stokes equations). The averaged governing equations contain additional unknown variables, and turbulence models are needed to determine these variables in terms of known quantities. Therefore, with this averaging 
approach the turbulence is modeled and only the unsteady, mean flow structures that are primarily larger than the turbulent eddies are resolved. This is the main difference with the other two widely used numerical approaches for turbulent flows, known as DNS (Direct Numerical Simulation) and LES (Large Eddy Simulation). In DNS, the Navier-Stokes equations are applied and solved directly without the application of a turbulence model, resolving the whole range of turbulent eddies. In LES on the other hand, large eddies are resolved directly, while small eddies are modeled. DNS and LES may provide detailed information on turbidity current flows but their major disadvantage is that their application is limited due to large computational requirements. On the other hand, RANS may not provide detailed information from a microscopic point of view, but is quite accurate and attractive for modeling large scale, three dimensional flows of practical engineering interest due to the relatively low computational cost.

In the simulations of the current work the Renormalization-group (RNG) $k$ - $\varepsilon$ model is applied for turbulence closure. This model was derived using a rigorous statistical technique, the renormalization group theory. The basic form of the RNG $k-\varepsilon$ model is similar to the standard $k-\varepsilon$ model, but it includes a number of refinements, rendering it more appropriate for the numerical simulations of the present investigation, as it is more accurate for swirling flows and rapidly strained flows and also accounts for low Reynolds number effects. Moreover, it provides an analytical formula for the calculation of the turbulent Prandtl numbers. At this point it should be mentioned that the RNG $k-\varepsilon$ model is also modified accordingly in order to simultaneously account for the primary (continuous) phase and the secondary (dispersed) phases of the simulated flows. This modification in FLUENT is based on a number of assumptions. In more detail, turbulent predictions for the continuous phase are obtained using the RNG $k-\varepsilon$ model, supplemented with extra terms that include the interphase turbulent momentum transfer. Predictions for turbulence quantities for the dispersed phases are obtained using the Tchen theory of dispersion of discrete particles by homogeneous turbulence [29]. Interphase turbulent momentum transfer is also assumed, in order to take into account the dispersion of the secondary phases transported by the turbulent fluid motion. Finally, a phase-weighted averaging process is assumed, so that no volume fraction fluctuations are introduced into the continuity equations [28].

\subsection{Governing Equations}

The volume of phase $q, V_{q}$ is defined by the following relationship [28]: 


$$
V_{q}=\int_{V} a_{q} d V
$$

where,

$$
\sum_{q=1}^{n} a_{q}=1
$$

and $a_{q}$ is the volume fraction of phase $q$.

The effective density of phase $q$ is:

$$
\hat{\rho}_{q}=a_{q} \rho_{q}
$$

where $\rho_{q}$ is the physical density of phase $q$.

The continuity, the fluid-fluid, and fluid-solid momentum equations that are actually solved by the model are described by equations (4), (5) and (6) respectively, for the general case of an $n$ - phase flow consisting of granular and non-granular secondary phases [28]:

$$
\begin{gathered}
\frac{1}{\rho_{r q}}\left(\frac{\partial}{\partial t}\left(a_{q} \rho_{q}\right)+\nabla \cdot\left(a_{q} \rho_{q} \vec{v}_{q}\right)=0\right) \\
\frac{\partial}{\partial t}\left(a_{q} \rho_{q} \vec{v}_{q}\right)+\nabla \cdot\left(a_{q} \rho_{q} \vec{v}_{q} \vec{v}_{q}\right)=-a_{q} \nabla p+\nabla \cdot \overline{\tau_{q}}+a_{q} \rho_{q} \vec{g}+ \\
\sum_{p=1}^{n}\left(K_{p q}\left(\vec{v}_{p}-\vec{v}_{q}\right)\right)+\left(\vec{F}_{q}+\vec{F}_{l i j t, q}+\vec{F}_{v m, q}\right) \\
\frac{\partial}{\partial t}\left(a_{s} \rho_{s} \vec{v}_{s}\right)+\nabla \cdot\left(a_{s} \rho_{s} \vec{v}_{s} \vec{v}_{s}\right)=-a_{s} \nabla p-\nabla p_{s}+\nabla \cdot \overline{\tau_{s}}+a_{s} \rho_{s} \vec{g}+ \\
\sum_{l=1}^{N}\left(K_{l s}\left(\vec{v}_{l}-\vec{v}_{s}\right)\right)+\left(\vec{F}_{s}+\vec{F}_{l i j t, s}+\vec{F}_{v m, s}\right)
\end{gathered}
$$

where $\rho_{r q}$ is the phase reference density, or the volume averaged density of the $q^{\text {th }}$ phase in the solution domain, $\overrightarrow{v_{q}}$ is the velocity of phase $q, \vec{v}_{p}$ is the velocity of phase $p, p$ is the pressure shared by all phases, $\overline{\overline{\tau_{q}}}$ is the $q^{\text {th }}$ phase stress-strain tensor, $\vec{g}$ is the gravitational acceleration, $K_{p q}$ is the interphase momentum exchange coefficient, $\vec{F}_{q}$ is an external body force (e.g. gravity), $\vec{F}_{l i f t, q}$ is a lift force and $\vec{F}_{v m, q}$ is a virtual mass force. $K_{l s}=K_{s l}$ is the momentum exchange coefficient between fluid phase $l$ and solid phase $s$ and $N$ is the total number of phases. The stress-strain tensors $\overline{\overline{\tau_{q}}}$ and $\overline{\overline{\tau_{s}}}$ are calculated by the following relationships: 


$$
\begin{aligned}
& \overline{\overline{\tau_{q}}}=a_{q} \mu_{q}\left(\nabla \overrightarrow{v_{q}}+\nabla \vec{v}_{q}^{T}\right)+a_{q}\left(\lambda_{q}-\frac{2}{3} \mu_{q}\right) \nabla \cdot \overrightarrow{v_{q}} \bar{I} \\
& \overline{\overline{\tau_{s}}}=a_{s} \mu_{s}\left(\nabla \overrightarrow{v_{s}}+\nabla \vec{v}_{s}^{T}\right)+a_{s}\left(\lambda_{s}-\frac{2}{3} \mu_{s}\right) \nabla \cdot \overrightarrow{v_{s}} \bar{I}
\end{aligned}
$$

where $\mu_{q}$ and $\mu_{s}$ are the shear viscosities of phases $q$ and $s, \lambda_{q}$ and $\lambda_{s}$ are the bulk viscosities of phases $q$ and $s$, and $\overline{\bar{I}}$ is the identity tensor.

The momentum exchange between the various phases involved in a multiphase flow is based in the value of the interphase exchange coefficients. Therefore, these coefficients are very important for the simulation of granular multiphase flows, as turbidity currents. In the simulations of the present paper the fluid-solid momentum exchange coefficient, between the ambient water (primary phase) and the suspended sediment particles (secondary phase) is calculated using the Syamlal-O'Brien model [28], which is based on measurements of the terminal velocities of particles in fluidized or settling beds. This model was selected for the simulations of the present investigation, as a series of trial numerical runs indicated that this gives the best results, in comparison with the corresponding experimental measurements, both for the lock-gate and the loose bed cases.

As it can be seen from Equation (6), in the case of granular flows, in the regime where the solids volume fraction is less than its maximum allowed value, a solids pressure is calculated independently and used for the pressure gradient term $\left(\nabla p_{s}\right)$, in the fluid-solid momentum equation. This solids pressure is composed of a kinetic term as well as a second term due to particle collisions and is calculated using the following relationship [28]:

$$
p_{s}=a_{s} \rho_{s} \Theta_{s}+2 \rho_{s}\left(1+e_{s s}\right) a_{s}^{2} g_{0, s s} \Theta_{s}
$$

where $e_{s s}$ is the coefficient of restitution for particle collisions, $g_{0, s s}$ is the radial distribution function, and $\Theta_{s}$ is the granular temperature which is proportional to the kinetic energy of the fluctuating particle motion. Trial numerical simulations indicated that the solids pressure is significant at various regions and stages of the simulated flows. Therefore, this additional pressure was taken into consideration in the numerical simulations presented in the present paper.

The effect of lift forces in the secondary phase solid particles is also taken into account, in the calculations of the present investigation. These lift forces act on particles mainly due to velocity gradients in the primary-phase flow field. The lift force will be more significant for larger particles. A main assumption is that the particle diameter is much 
smaller than the interparticle spacing. Hence, the inclusion of lift forces is not appropriate for closely packed particles or for very small particles. The lift force acting on a secondary phase $p$ in a primary phase $q$ is calculated in FLUENT, using the following equation [28]:

$$
\vec{F}_{l i f t}=-C_{L} \rho_{q} a_{p}\left(\vec{v}_{q}-\vec{v}_{p}\right) \times\left(\nabla \times \vec{v}_{q}\right)
$$

where $C_{L}$ is the lift coefficient. For the numerical runs considered in the present paper, values of the lift coefficient ranging from 0.1 to 0.5 gave the best results in comparison with the corresponding experimental measurements, in each case.

The virtual mass force is usually significant, in cases where the secondary phase density is much smaller than the primary phase density [28]. For example, the virtual mass force would be significant in the case of air bubbles moving through water, as in this case the density of air is much smaller than the density of the ambient water and the added mass (by the surrounding water) in the air bubbles would be much larger than their own mass. In all of the turbidity current cases considered in the present paper, the secondary phase density (solid particles) is larger than the primary phase density (fresh water). However, these densities are of the same order of magnitude and recent works have indicated that the virtual mass force in such cases may become important in non-dilute mixtures. Despite these recent findings, the virtual mass force is not taken into consideration in the calculations of the present paper. However, the importance of the virtual mass force will be tested in future works, conducting additional simulations where the virtual mass force is included in the calculations.

Other forces, such as the Faxen and Magnus forces are not taken into consideration. The Faxen force is very small, as it scales with $\left(d_{p} / l\right)^{2}[30]$, where $d_{p}$ is the diameter of the particles and $l$ is a characteristic length scale of the flow. Therefore, the Faxen forces are disregarded in the numerical simulations of the present paper. The Magnus force is significant in cases where the particle Reynolds number $\left(\operatorname{Re}_{p}=d_{p}\left(v_{p}-v_{f}\right) / v_{f}\right.$, where $v_{p}$ is the particle velocity, $v_{f}$ is the fluid velocity and $v_{f}$ is the kinematic viscosity of the fluid) is greater than 1 [31]. In all cases considered in the present paper the particle Reynolds number is much smaller than 1 and therefore, the Magnus force effect is disregarded.

The general transport equations for the turbulence kinetic energy $k$ and the turbulence dissipation rate $\varepsilon$, of the RNG $k-\varepsilon$ turbulence model, can be described by equations (11) and (12) respectively [28]:

$$
\frac{\partial}{\partial t}(\rho k)+\frac{\partial}{\partial x_{i}}\left(\rho k u_{i}\right)=\frac{\partial}{\partial x_{j}}\left(a_{k} \mu_{e f f} \frac{\partial k}{\partial x_{j}}\right)+G_{k}+G_{b}-\rho \varepsilon
$$




$$
\begin{aligned}
& \frac{\partial}{\partial t}(\rho \varepsilon)+\frac{\partial}{\partial x_{i}}\left(\rho \varepsilon u_{i}\right)=\frac{\partial}{\partial x_{j}}\left(a_{\varepsilon} \mu_{e f f} \frac{\partial \varepsilon}{\partial x_{j}}\right)+ \\
& C_{1 \varepsilon} \frac{\varepsilon}{k}\left(G_{k}+C_{3 \varepsilon} G_{b}\right)-C_{2 \varepsilon} \rho \frac{\varepsilon^{2}}{k}-R_{\varepsilon}
\end{aligned}
$$

where $u$ represents velocity, $\rho$ is the local mixture density, $G_{k}$ is the generation of turbulence kinetic energy due to mean velocity gradients, $G_{b}$ is the generation of turbulence kinetic energy due to buoyancy, $a_{k}$ and $a_{\varepsilon}$ are the inverse effective Prandtl numbers for $k$ and $\varepsilon$ respectively, $\mu_{e f f}$ is the effective viscosity and $C_{1 \varepsilon}, C_{2 \varepsilon}$ and $C_{3 \varepsilon}$ are turbulence model constants. The term $R_{\varepsilon}$ in the $\varepsilon$ equation accounts for the effects of rapid strain and streamline curvature.

As it can be seen from Equation (11) turbulence kinetic energy tends to be augmented $\left(G_{b}>0\right)$ in cases of unstable stratification. For stable stratification buoyancy tends to suppress the turbulence $\left(G_{b}<0\right)$. In the RNG $k-\varepsilon$ model in FLUENT, the effect of buoyancy on the generation of $k$ is always included when a non-zero gravity field is specified. The degree to which the dissipation of turbulent kinetic energy $(\varepsilon)$ is affected by the buoyancy is determined by the constant $C_{3 \varepsilon}$, which is not a user defined value. Instead, it is calculated by the following relationship:

$$
C_{3 \varepsilon}=\tanh \left|\frac{v}{u}\right|
$$

where $v$ is the component of the flow velocity parallel to the gravitational vector and $u$ is the perpendicular one. Hence, $C_{3 \varepsilon}$ will become unity for buoyant shear regions where the main flow direction is aligned with the direction of gravity and zero for buoyant shear regions that are perpendicular to the gravitational vector [28].

\subsection{Solution Procedure}

The proposed numerical model solves the governing equations sequentially using the control-volume method. Hence, the equations are integrated about each control-volume, yielding discrete equations for the conservation of each quantity. An implicit formulation is used, in order for the discretized equations to be converted to linear equations for the dependent variables in every computational cell. Further details regarding the solution procedure can be found at FLUENT User's Guide [28]. 


\subsection{Boundary Conditions}

This subsection of the paper summarizes the general aspects and characteristics of the boundary conditions used in the numerical applications of the present paper.

At inlets, a velocity-inlet boundary condition is used. With this type of boundary condition, a uniform distribution of all the dependent variables is prescribed at the face representing the sediment laden water inflow. In more detail, the velocity magnitudes of the primary and secondary phases with directions normal to the inlet face are specified, assuming constant, uniform values. Moreover, the volume fractions of the secondary phases at the inlet are specified.

For the outlets, a pressure-outlet boundary condition is applied. Using this type of boundary condition, all flow quantities at the outlets are extrapolated from the flow in the interior domain. A set of "backflow" conditions can be also specified, allowing reverse direction flow at the pressure outlet boundary during the solution process. In other words, this type of outlet condition serves as an open flow boundary, allowing the flow to freely exit or enter the computational domain during the calculations.

At the free ambient water surfaces, a symmetry boundary condition is used, which is typically well above the turbidity current. Thus, there are neither convective nor diffusive fluxes across the top surface. This type of free surface boundary condition has also been used by other researchers in literature [20, 22]. Farrell and Stefan [32] have found that for a plunging reservoir flow, the relative error that can be introduced by this approximation of the free surface, is of the order of $10^{-3}$ and does not influence the velocity field.

The solid boundaries are specified as stationary walls with a no-slip shear condition. In cases that wall roughness effects need to be included in the calculations, the law-of-thewall modified for roughness is applied using the following relationship:

$$
\frac{u_{p} u_{*}}{t_{w} / \rho}=\frac{1}{\kappa} \ln \left(E \frac{\rho u_{*} y_{p}}{\mu}\right)-\Delta B
$$

where $u_{p}$ is the mean flow velocity at point $p, u_{*}$ is the friction velocity, $\tau_{w}$ is the wall shear stress, $\rho$ is the fluid density at the wall, $\mu$ is the dynamic viscosity, $\kappa$ is the von Karman constant, $E$ is an empirical constant having a value of 9.793, $y_{p}$ is the distance from point $\mathrm{p}$ to the wall and $\Delta B$ is a roughness function that in general depends on the wall roughness height, $K_{s}$. The roughness function is estimated using the formulas proposed by Cebeci and Bradshaw [33]. 
Turbulent flows are significantly affected by the presence of walls. Very close to the wall, viscous damping and kinematic blocking reduce the tangential and normal velocity fluctuations respectively. However, in the outer part of the near wall region, the turbulence is rapidly augmented by the production of turbulent kinetic energy due to the relatively large gradients in mean velocity.

In FLUENT, there are two different approaches for modeling the near wall region. In the first approach, the viscous sub-layer and the buffer sub-layer are not resolved. Instead, semi-empirical formulas known as "wall functions" are used in order to link the viscosity affected sub-layers between the wall and the fully-turbulent region. In other words, the use of wall functions obviates the need of turbulence models modification in order to account for the presence of walls and does not require the construction of very fine computational meshes at the near-wall regions. In the second approach, known as "near-wall modeling" approach, the turbulence models are modified in order for the viscosity affected near-wall regions to be resolved with a computational mesh all the way to the wall. However, the computational mesh must be significantly fine in these regions. This approach may require more computational effort, but it gives more accurate predictions at the near-wall region of the computational domain. Therefore, wall functions should only be used in cases where the complexity and size of the computational domain as well as the available computational resources, do not allow the construction of very fine meshes at the near-wall regions.

At this point it should be mentioned that these two different approaches for modeling the near wall region are both used in the applications of the present paper.

\section{Description of Numerical Simulations}

In order to verify in detail the proposed model, the published laboratory experiments conducted by Gladstone et al. [10] and Baas et al. [11] are reproduced numerically, and the results are compared aiming to evaluate how realistic and reliable the numerical simulations of the proposed model are. The first series of laboratory experiments consist of fixed-volume lock-gate releases of dilute mixtures containing two different sizes of silicon carbide particles. The second series of laboratory experiments consist of high-density sediment-water mixtures released with a quasi-steady rate, through a small inflow gate, into an inclined channel which is connected to a tank, were an expansion table covered with loose sediment is positioned. 


\subsection{Lock-gate Cases}

\subsubsection{Physical Problem}

The basic experimental set-up is shown in Figure 1, while the relative initial proportions, of each particle size grade used in the experimental runs conducted by Gladstone et al. [10], are summarized in Table1. As it can be seen, the experimental set-up consists of a glass flume tank $5.7 \mathrm{~m}$ long and $0.2 \mathrm{~m}$ wide, filled with tap water at a depth of $0.4 \mathrm{~m}$. A gate was situated $0.2 \mathrm{~m}$ from one end, providing a lock volume of $0.016 \mathrm{~m}^{3}$. The particles were placed in the lock volume and stirred vigorously in order to ensure full particle suspension. The stirrer was removed and each experiment was initiated by lifting the lock-gate after $2 \mathrm{sec}$, in order to reduce the turbulence level of the suspension. Silicon carbide particles with a density of $3,217 \mathrm{~kg} / \mathrm{m}^{3}$ were used to create the dilute mixtures. Two different size fractions of particles were used, with average particle diameters of $25 \mu \mathrm{m}$ and $69 \mu \mathrm{m}$ respectively. The total mass of particles used in each experiment was $180 \mathrm{~g}$, suspended in approximately 161 of tap water, yielding a volumetric fraction of $3.49 \times 10^{-3}$ and a current density of $1,005.9 \mathrm{~kg} / \mathrm{m}^{3}$. As it can be seen from Table 1, seven experimental runs were conducted. The only variable altered between each run was the initial volumetric concentration of each particle size fraction.

\subsubsection{Application of numerical Model}

In the numerical simulations reproducing the laboratory experiments described above, the same initial conditions as in the experiments were used for all cases, assuming uniform particle size fractions of $25 \mu \mathrm{m}$ and $69 \mu \mathrm{m}$ respectively (Table 1). The numerical runs are named in accordance with the experimental ones for comparison purposes. The computational geometry and mesh used in the simulations as well as the applied boundary conditions are illustrated in Figure 2. The computational mesh used in the simulations comprises with a total number of 68,950 hexahedral cells. Two different cell zones were used. The first zone corresponds to the flume part that is initially filled with tap water while the second zone corresponds to the lock volume that initially contains the dilute water-particle mixtures. In both zones, grid clustering was used near the bottom boundary. For the first layer of cells a height of $2 \mathrm{~mm}$ from the bottom boundary was used while the height of the succeeding layers was gradually increased with a growth rate of 1.05 . The cell dimensions in the $\mathrm{X}$ and $\mathrm{Y}$ directions was kept constant having a value of $3 \mathrm{~cm}$. Sensitivity tests were performed with 
similar computational meshes consisting of different number of cells in order to ensure that the solution is mesh independent. In Figure 3, the flow front position versus time is depicted for three different meshes, in the case of numerical Run A. The first mesh is the one used in the simulations presented in the present paper, the second is a coarser mesh consisting of 25,020 cells and the third is a finer one consisting of 103,968 cells. The calculation time step was in each case adjusted accordingly to the cell size characteristics of each mesh. As it can be seen the resulting graphs compare very well and hence the numerical solutions can be considered mesh-independent. In more detail, comparing the results of the coarser and the finer mesh it is found that by increasing the number of cells by a factor of 4.15 , the average difference in the values of the distance travelled by the front with respect to time, is calculated to be only $2.5 \%$. At this point it should be mentioned that minimal differences also resulted, by comparing velocity time series at various points in the computational domain as well as contours of water-sediment mixture density at fixed times, in ZX planes at $\mathrm{Y}=0 \mathrm{~m}$.

The general characteristics of the applied boundary conditions indicated in Figure 2, have been described in the previous section of the paper (Section 2). However, it should be mentioned that for the bottom wall boundary of the domain in the lock-gate numerical runs, the "enhanced wall treatment" which is a near-wall modeling approach is used, for the prediction of the turbulent quantities at the near-wall region. The geometry of the computational domain in this case is simple and straight-forward allowing the use of a computational mesh consisting of hexahedral cells. With this type of mesh, the height of the computational cells can be considerable smaller than the breadth and length of the cells. This allows the use of a very fine mesh near the bottom wall boundaries, without a significant increase in the total number of cells and therefore in the computational effort required for the simulations, allowing the application of the enhanced wall treatment approach.

\subsection{Loose Bed Cases}

\subsubsection{Physical Problem}

The basic experimental set-up for the loose bed experiments [11] is shown in Figure 4, while the starting conditions for experimental runs $1,3,4,7,8,9,13$ and 14 that are reproduced numerically in the present paper are summarized in Table 2. As it can be seen, the experimental set-up consists of a straight inclined channel that leads in a water tank, where an expansion table is positioned. The channel and tank are both filled with fresh water. At the upstream end of the channel, a mixing tank is positioned where mixing of fresh water with 
sediment occurs. The water-sediment mixture is released in the channel, through a small inflow gate at the bottom part of the front boundary of the mixing tank. The inflow gate has a height of $0.035 \mathrm{~m}$ and a width of $0.18 \mathrm{~m}$, giving a cross-sectional area of $0.0063 \mathrm{~m}^{2}$. In all experimental runs, the expansion table is covered initially with a $0.03 \mathrm{~m}$ thick layer of loose sediment of the same type and grain size, as the sediment released from the mixing tank. Water- sediment mixtures with fine sand, coarse silt and very fine sand are released from the gate at various initial concentrations, channel inclinations and discharges, while in some of the runs the channel bed is roughened with fine sand particles glued uniformly at the bottom of the channel (Table 2). During the experimental runs, a video window was used for monitoring the turbidity currents in the channel. Quasi-steady discharge is maintained for the first 15 seconds after the gate release, and for the next 15 seconds the sediment-water mixture discharge gradually reduces.

\subsubsection{Application of numerical Model}

In the numerical simulations reproducing the laboratory experiments described above, a constant inflow condition is used, that corresponds to the 15 -second slurry discharge from the mixing tank. In other words, in all numerical runs the first 15 seconds of quasi-steady discharge from the laboratory runs is considered for comparison purposes. The gradually reducing part of the discharge is not simulated numerically. This is because no details regarding these reducing flow rates are given in the paper of Baas et al. [11]. Since the channel inclinations used in the experimental runs were $3.7^{\circ}$ and $8.6^{\circ}$, two different geometries were constructed for the corresponding numerical simulations. The computational geometry and mesh used in the simulations as well as the applied boundary conditions are illustrated in Figure 5, for the case with the $8.6^{\circ}$ channel slope. In the case of $3.7^{\circ}$ channel slope these features are similar.

In the geometry with $8.6^{\circ}$ channel slope, the mesh is comprised with a total number of 398,048 tetrahedral cells, while in the geometry with $3.7^{\circ}$ channels slope the mesh is comprised with a total number of 440,058 tetrahedral cells. In both cases two different cell zones were used. The first zone corresponds to the $0.03 \mathrm{~m}$ thick, horizontal layer of loose sediment, which lies on the expansion table in the laboratory experiments, consisting of cells with an edge size of $0.03 \mathrm{~m}$. In the second zone, comprised by the inclined channel and the volume above the loose sediment layer, grid clustering was used near the bottom, starting with cells having an edge dimension of $0.03 \mathrm{~m}$ and using a growth rate of 1.2 away from the bottom boundaries, in all directions. This technique is used in order to reduce the total number 
of cells within the computational domain, by using fine mesh in regions of interest which gradually coarsens approaching regions were no great detail is required. Since it is expected that the simulated turbidity currents will form and evolve at a layer close to the bottom boundaries of the domain, fine mesh is used close to these boundaries that gradually coarsens away from them. Hence, as it can be observed the mesh looks coarse at the top boundaries of the domain, but this do not influence the solution, as the simulated currents are formed in the vicinity of the bottom boundaries of the domain were the mesh resolution is adequately fine. Sensitivity tests were performed with similar computational meshes, consisting of different number of cells, in order to ensure that the solution is mesh-independent. In Figure 6, the flow front position versus time is depicted for three different meshes, in the case of Run 1 . The first mesh is the one used in the simulations presented in the present paper $(398,048$ cells), the second is a coarser mesh consisting of 209,819 cells and the third is a finer one consisting of 737,382 cells. The calculation time step was in each case adjusted to the cell size characteristics of each mesh. As it can be seen, the resulting graphs compare very well and therefore the numerical solutions can be considered mesh-independent. In more detail, comparing the results of the coarser and the finer mesh it is found that by increasing the number of cells by a factor of 3.5, the average difference in the values of the distance travelled by the front with respect to time, is calculated to be only $2.4 \%$. At this point it should be mentioned that minimal differences also resulted, by comparing velocity time series at various points in the computational domain as well as contours of water-sediment mixture density at fixed times, in ZX planes at $\mathrm{Y}=0 \mathrm{~m}$.

The characteristics of the applied boundary conditions indicated in Figure 5, have been described in the previous section of the paper (Section 2). However, it should be mentioned that for the bottom wall boundary of the domain in the loose bed numerical runs, the "standard wall functions" are used, for the prediction of the turbulent quantities at the near-wall region. The computational domain in the loose bed cases has a much more complicated geometry, where the construction of a computational mesh consisting of hexahedral cells is very difficult. Therefore, a computational mesh consisting of tetrahedral cells is used. This type of mesh does not allow large differences in the length of the edges comprising each computational cell. Therefore, the use of very fine mesh close to the bottom boundaries is not feasible as this would significantly increase the total number of cells required for the calculations. Therefore, the enhanced wall treatment is not feasible in this case (due to limitations in the available computational resources) and the standard wall functions approach is used instead. 
As it can be seen from Figures 4 and 5, the outlet boundaries in the numerical geometry are positioned at the ends of the expansion table. The solid downstream walls of the tank, where the expansion table was positioned in the experiments, are not taken into consideration since their position is outside of the computational domain. Moreover, it should be mentioned that since the non-reflecting boundary condition option is not available with the pressure-based solver that is used in the simulations of the present paper, the solution was in each case checked for possible spurious reflections at the downstream, open-flow boundaries. For this purpose each run was continued for additional 55 seconds, in order for the simulated turbidity currents to reach and pass through these boundaries. In all cases the simulated turbidity currents exit the flow domain at the downstream boundaries without any observed reflections.

In order to account for the mixing and interaction of the sediment particles between the loose bed layer and the expanding turbidity current in direct analogy with the experiments, before the beginning of the calculations, during the initialization of the solution, the primary phase (fresh water) is assigned in the computational domain. Afterwards the secondary phase (sand particles) with a volume fraction of 0.56 is patched in cell zone 1 (Figure 5). Hence, before the beginning of the calculations, cell zone 1 contains sand particles with a volume fraction of 0.56 while cell zone 2 contains fresh water. Cell zones 1 and 2 are connected at their interface, without any boundary condition applied at this interface. Therefore, for the loose bed numerical simulations the bottom boundary of the inclined channel and the bottom boundary of the tank where the loose sediment layer lies (bottom of cell zone 1) are stable solid walls with a zero-flux condition (as in the laboratory experiments) and the loose sediment layer that initially lies on the stable bottom boundary of the tank, can freely interact and mix with the expanding turbidity currents, through the interface of cell zones 1 and 2. In this way the scoured regions of the loose sediment layer can be identified after the passage of the expanding turbidity currents.

The initial conditions for all the numerical runs are the same as the corresponding experimental runs (Table 2) and the numerical runs are named in accordance with the experimental ones for comparison purposes. 


\section{Comparison of Numerical Simulations with Experiments}

\subsection{Lock-gate Cases}

In this subsection of the paper, specific quantitative results of the current front speed that are presented in the laboratory work of Gladstone et al. [10], are compared with the corresponding numerical results, in order to validate the reliability of the numerical simulations for the lock-gate cases. Since the laboratory work of Gladstone et al. [10] does not present any vertical velocity profiles of the experimentally simulated lock-gate currents, the non-dimensional vertical profiles of the streamwise velocity component for numerical runs A (100\% fine particles), D (50\% fine and 50\% coarse particles) and G (100\% coarse particles) are constructed and compared with dimensionless experimental data from the laboratory work of Garcia [12], in order to ensure that the numerically predicted profiles fall within the scatter range of analogous experimental data that are reported in the literature.

\subsubsection{Front Speed}

Front speed is one of the most studied parameters for lock-exchange turbidity currents. Figure 7 compares the simulated and observed current front position versus time for all the lock-gate cases considered in the present paper. As it can be seen, in general the numerical simulations show a good match with the experimental data, adequately predicting the differences in the flow front advance among the generated currents with respect to the different relative proportions of coarse and fine particles that were used in the initial suspensions. Furthermore, in accordance with the experiments, all the resulting numerical curves consist of an initial steep section approximately up to the first $20 \mathrm{sec}$ that gradually becomes less steep thereafter. The initial steep region corresponds to the slumping phase of the turbidity currents, where the flow front velocity is approximately constant. The gradual reducing speed in the succeeding section of the curves is due to the continuous particle deposition and the associated decrease in the excess density which is the driving force of the flows. This indicates that the numerical model successfully captures the initial slumping phase of the currents (constant speed regions) as well as the different deposition rate between each run (gradually reducing speed regions). The observed divergence between the experimental and the numerical curves at various times, might be partially attributed to possible over-estimation or under-estimation of the flow front position in the particular laboratory runs, due to the difficulty in the visual definition of the exact flow front position, 
since these laboratory difficulties are stated in the work of Gladstone et al. [10]. Another possible reason for the observed divergence might be the overall assumptions in the numerical simulations (e.g. uniform grain size in each particle class).

In Figure 8 the simulated and observed current front position versus initial percentage of coarse fraction for all the lock-gate cases, at two fixed times (72 and $111 \mathrm{sec}$ ), are compared. As it can be seen, the numerical model successfully predicts the corresponding flow front position measured in the experimental runs, in each case. Again, the differences between the experimental and numerical values for some of the runs could be attributed to the difficulties in the definition of the exact flow front position during the experiments.

\subsubsection{Vertical Structure}

In order to examine the validity of the vertical structure of the simulated lock-gate cases, the non-dimensional vertical profiles of the streamwise velocity component for numerical runs $\mathrm{A}$ and $\mathrm{D}$ are constructed and compared with analogous dimensionless experimental data from the laboratory work of Garcia [12]. For each of the numerical runs, the profiles are taken at time $\mathrm{t}=30 \mathrm{sec}$, from the body of the currents, at a distance $\mathrm{X}=80 \mathrm{~cm}$ from the lock-gate position. The velocity values are normalized with the depth-averaged velocity $U$ while the vertical distances are normalized with the turbidity current thickness $h$, defined as follows:

$$
\begin{gathered}
U=\frac{\int_{0}^{\infty} u^{2} d z}{\int_{0}^{\infty} u d z} \\
h=\frac{\left(\int_{0}^{\infty} u d z\right)^{2}}{\int_{0}^{\infty} u^{2} d z}
\end{gathered}
$$

where $u$ is the streamwise velocity and $z$ is the vertical distance above the bed of the flume. In each of the three cases the bulk densimetric Froude number of the flows was less than one. Therefore the simulated dimensionless velocity profiles are compared with experimental results for subcritical flows. The bulk densimetric Froude number was calculated using the following relationship:

$$
F r_{d}=\frac{U}{\sqrt{R g C h}}
$$

where $\mathrm{g}$ denotes the gravitational acceleration, $\mathrm{R}$ denotes the submerged specific gravity of the sediment particles and $\mathrm{C}$ denotes the layer-averaged, volume concentration of sediment in 
the turbidity current. The following formulas were used for the calculation of $\mathrm{R}$ and $\mathrm{C}$ :

$$
\begin{gathered}
R=\left(\rho_{\text {sediment }}-\rho_{\text {fluid }}\right) / \rho_{\text {fluid }} \\
C=\frac{\int_{0}^{\infty} u c}{U h}
\end{gathered}
$$

where $\rho_{\text {sediment }}$ is the density of the sediment particles, $\rho_{\text {fluid }}$ is the density of the ambient fluid and $c$ is the sediment volume fraction. The numerical profiles and the corresponding experimental data are compared in Figure 9. As it can be seen, the numerically predicted dimensionless profiles fall within the general scatter range of the dimensionless data for subcritical currents that resulted from the laboratory experiments of Garcia [12]. Therefore, it can be concluded that the numerical model gives fairly reasonable predictions regarding the vertical structure of the simulated currents.

\subsection{Loose Bed Cases}

In this subsection of the paper, specific macroscopic observations and quantitative results that are described and presented in the laboratory work of Bass et al. [11], are compared with the corresponding numerical observations and results, in order to validate the reliability of the numerical simulations for the loose bed cases. Since the laboratory work of Baas et al. [11] does not present any vertical velocity profiles of the experimentally simulated loose bed currents, the non-dimensional vertical profiles of the streamwise velocity component for numerical runs 1 (fine sand), 7 (coarse silt) and 14 (very fine sand) are constructed and compared with dimensionless experimental data from the laboratory work of Garcia [12], in order to ensure that the numerically predicted profiles fall within the scatter range of analogous experimental data that are reported in the literature.

\subsubsection{Macroscopic Observations}

The most important macroscopic observations regarding the flow structure and evolution of the experimental runs that are discussed in the laboratory work of Baas et al. [11] are summarized below:

- The head of the currents is continuously supplied by faster moving fluid from the body of the flow.

- In the video window position, two distinct layers are formed in the body of the turbidity currents. A lower dense layer, moving parallel to the bed, and an 
upper more dilute layer, where mixing with the ambient fluid takes place.

- At the entrance to the expansion table, the expansion of the current is accompanied by the development of an internal hydraulic jump.

- Downstream of the hydraulic jump, the turbidity current spreads out radically in all directions, with the presence of a prominent head, forming a channellevee system that ends with a depositional lobe.

These qualitative experimental observations are common to all the experimental runs, regardless the initial sediment concentration and type, the water-sediment mixture discharge, the slope and roughness of the channel (Table 2). Figures 10-14 indicate that the macroscopic observations discussed in the paper by Baas et al. [11] are evident in the numerical runs as well. In accordance to the experimental observations, these general qualitative results are also common in all of the numerical runs conducted. Therefore, only Run 1 has been chosen in these figures for illustration purposes.

In Figure 10, contours of velocity magnitude for time $\mathrm{t}=5 \mathrm{sec}$ are plotted, in a ZX section at $\mathrm{Y}=0 \mathrm{~m}$. As it can be seen, within the current two major velocity layers are formed. The first layer is located at the bottom of the flow encountering velocity values greater than $0.80 \mathrm{~m} / \mathrm{sec}$. In the streamwise direction this layer extends approximately up to the back of the turbidity current head. The second layer is a slower moving fluid layer that lies on top of the first layer, with velocity values ranging from 0 to $0.80 \mathrm{~m} / \mathrm{sec}$. The formation of these layers clearly demonstrates that the head of the turbidity current is continuously supplied by a faster moving fluid from the body of the current, in agreement with the experimental observations.

For flow time $t=7 \mathrm{sec}$, the head of the numerically simulated turbidity current has just passed the downstream end of the region, corresponding to the video window used in the laboratory set-up. Figure 11 illustrates the contours of fine sand volume fraction in a ZXsection at $\mathrm{Y}=0 \mathrm{~m}$, for this time step. It is evident that two distinct concentration layers are formed, in agreement with the experimental observations. The first one is a dense layer with sediment volume fraction values ranging from 0.13 to 0.04 , which is positioned at the bottom of the turbidity current parallel to the channel bed. The second one is a more dilute layer that lies on top of the dense layer, with sediment volume fraction values ranging from 0.04 to 0.01 . These distinct concentration layers are in direct analogy to the distinct velocity layers illustrated previously in Figure 10. The driving force (excess density) of the dense bottom layer is greater than this of the dilute layer at the top of the current. Therefore, the dense layer moves faster than the dilute layer. The instabilities observed at the top of the slower moving dilute layer in Figure 10, which roughly coincide with the interface between the turbidity 
current and the ambient water, clearly indicate that the considerable mixing with the ambient fluid that was observed in the experiments at the top of the dilute layer, is also evident in the numerical results. This type of mixing at the interface occurs due to the entrainment of the ambient water into the turbidity current. These instabilities as well as the observed backward moving flux from the head of the current (Figure 10), are a result of vortex shedding induced by the mixing of the current with the ambient water, which is an important aspect of turbidity current flows.

As observed in the experiments, the initiation and time evolution of the internal hydraulic jump, which is formed at the entrance of the numerically simulated turbidity current to the expansion tank, is illustrated in Figure 12. The black line that separates the grey colored area from the white domain corresponds to the interface between the particle laden water and the ambient water. As it can be seen, after the entrance of the generated turbidity current into the expansion tank, a hydraulic jump is clearly formed (time $\mathrm{t}=9 \mathrm{sec}$ ). The reason for the formation of the hydraulic jump can be attributed to the sudden slope change as well as to the entrainment of particles from the loose sediment layer that initially covers the expansion table, into the expanding turbidity current.

Finally, from Figure 13 it is evident that after the entrance to the expansion table, the numerically simulated turbidity current front spreads out radically in all directions, causing considerable erosion at the middle of the expansion table. At the sides of the eroded region two major current parts are observed that connect the expanding front with the feeding part of the flow in the channel. This type of expansion indicates that downstream of the hydraulic jump initiation point, the expansion of the turbidity current shows a tendency to form a channel-levee system (eroded region enclosed between side parts of expanding current) that ends with a depositional lobe (radically expanding turbidity current front), in agreement with the experimental observations.

\subsubsection{Quantitative Results}

In more detail, from a quantitative point of view, the flow properties of both the experimental and the numerical turbidity currents are quite different, for various values of initial suspended sediment concentration. Table 3, summarises the experimental and numerical values for turbidity current mean height, as well as, the thickness of the bottom dense layer at the position of the video window, for the various initial concentrations in the case of the fine sand suspensions (numerical runs 1, 3 and 4). As it can be seen, the numerical values are very close to the experimental ones. In the experiments the turbidity current mean 
height and the dense layer thickness are measured visually, at the video window, at the end of the quasi-steady discharge period that approximately corresponds to flow time $t=15 \mathrm{sec}$. For the numerical runs, the turbidity current height is calculated using Equation (16) at a vertical velocity profile taken at time $\mathrm{t}=15 \mathrm{sec}$ and distance $\mathrm{X}=2.5 \mathrm{~m}$, which corresponds approximately to the middle of the video window position, in order to satisfy an analogy with the experimental measurements. Accordingly, the thickness of the turbidity current dense layer in the numerical runs is estimated as the height, at which the corresponding vertical concentration profile, suddenly changes slope. These velocity and concentration profiles for runs 1, 2 and 4 are shown in Figures 14 (a) and 14 (b) respectively. The small differences between the experimental and numerical data in Table 4 could be partially attributed to the overestimation or underestimation of the corresponding interfaces in the laboratory measurements, due to difficulties in their visual definition, as these laboratory difficulties are stated in the work of Baas et al. [11]. Another possible reason for the differences might be the overall assumptions in the numerical simulations (uniform suspended sediment grain size, steady inflow etc).

The relationship between head velocity and initial suspended sediment concentration for fine-sand, very-fine sand and coarse silt laden turbidity currents is depicted in Figure 15, both for the numerical and the corresponding experimental runs (Runs 1, 3, 4, 7, 8, 13 and 14). In the laboratory experiments the head velocities were measured within the video window, using the video recordings. For direct analogy with the experimental runs the corresponding head velocities in the numerical runs were calculated by dividing the distance travelled by each front with the time required in each case, within the video window region. Once again, the numerical values are very close to the corresponding experimental values. Moreover, it is evident that the numerical model captures the same trend in the head velocity variation with respect to the increase of the initial suspended sediment concentration, in comply with the experimental runs. As it can be generally observed by the resulting curves, an increase in the initial suspended sediment concentration causes an increase in the front propagation velocity.

\subsubsection{Vertical Structure}

In order to examine the validity of the vertical structure of the simulated loose bed cases, the non-dimensional vertical profiles of the streamwise velocity component for numerical runs 1, 7 and 14 are constructed and compared with corresponding dimensionless 
experimental data from the laboratory work of Garcia [12]. For each of the numerical runs, the profiles are taken at time $\mathrm{t}=45 \mathrm{sec}$, from the body of the currents, at a distance $\mathrm{X}=3.3 \mathrm{~m}$ from the inflow-gate position. It should be mentioned that these runs were continued from time $\mathrm{t}=15$ to time $\mathrm{t}=45 \mathrm{sec}$ in order for the turbidity current front to exit the flow domain and quasi steady state to be reached at the position of the vertical profiles. In these cases the calculated, bulk densimetric Froude numbers are greater than unity and hence the simulated dimensionless velocity profiles are compared with experimental results for supercritical flows. The numerical profiles and the corresponding experimental data are illustrated in Figure 16. As it can be seen, the numerically predicted dimensionless data fall within the scatter range of the dimensionless data for subcritical currents that resulted from the laboratory experiments of Garcia [12]. However, at the near-wall region of the numerical profiles, a sharp change is observed in relation to the experimental values. This sharp change at the near-wall region could be attributed to the $3 \mathrm{~cm}$ mesh resolution that was used in the loose bed runs and the application of the standard wall functions that do not resolve but instead link the viscosity affected near-wall region with the fully turbulent outer region, though the use of empirically derived formulas. Since, this sharp change is not presented in the lock-gate cases (Figure 9), it can be concluded that the application of the enhanced wall treatment should be preferable at the bottom wall boundaries, in cases that the complexity and size of the computational domain geometry as well as the available computational resources, allow the construction of high-resolution meshes at the near-wall regions, since this provides more accurate and detailed predictions in the vicinity of the bottom wall boundaries.

\section{Further analysis of numerical simulations}

\subsection{Lock-gate Cases}

In this subsection of the paper, the temporal evolution of two extreme cases, from the numerical simulations reproducing the laboratory experiments of Gladstone et al. [10], is presented. The simulated time series of density contour and velocity vector plots for runs A (100\% fine) and G (100\% coarse), are illustrated in Figure 17, at 0, 5, 10 and $30 \mathrm{sec}$ after the release of the dilute mixtures. At $\mathrm{t}=0 \mathrm{sec}$ in both cases, the heavier fluid is locked on the left part of the flume. At $\mathrm{t}=5 \mathrm{sec}$ in each case, the denser fluid has already started to flow to the right along the bottom of the flume, while the lighter fluid flows to the left on top of the denser fluid, with the denser fluid having a slightly greater propagation speed than the lighter 
one. When the lighter fluid hits the wall on the left, a backflow wave is formed at the interface between the denser and the lighter fluids $(t=10 \mathrm{sec})$, due to reflection. This leads to a strong downward motion that drastically reduces the thickness of the turbidity current in the rear part of the flume. Up to $t=10 \mathrm{sec}$, the evolutions of the generated turbidity currents are similar for both runs. However, as the currents propagate further downstream $(\mathrm{t}=30 \mathrm{sec})$, the turbidity current generated in Run G (100\% coarse particles) travels at a lower speed (1.7 $\mathrm{m}$ in $30 \mathrm{sec})$, compared with the turbidity current generated in Run A $(2.2 \mathrm{~m}$ in $30 \mathrm{sec})$, with a much weakened head and a less defined tail. This is due to the larger particle deposition rates in Run $\mathrm{G}$ (particles with $69 \mu \mathrm{m}$ diameter) than in Run A (particles with $25 \mu \mathrm{m}$ diameter). The above observations are in qualitative agreement with the experimental and numerical simulation results reported by Necker et al. [17]. The different response of the generated currents with respect to the suspended particles diameter described here, in conjunction with the observations outlined in Subsection 4.1.1 of the present paper, clearly demonstrates that the suspended particle diameter as well as the amount of each particle size fraction in a mixture that contains various particle grain sizes, play a dominant role in the flow properties and dynamics of the generated turbidity currents.

\subsection{Loose Bed Cases}

In this subsection of the paper, some main results from the numerical simulations reproducing the experimental runs conducted by Baas et al. [11] are shown for various extreme cases, in order to indicate the differences in the flow dynamics for runs with different initial conditions and therefore identify the importance of various controlling parameters, such as the inflow velocity, the channel bed slope, the initial sediment concentration, the sediment grain size and the channel bed roughness, in the simulated turbidity currents.

The time evolution of suspended sediment volume fraction in a $3 \mathrm{D}$ view, for numerical Runs 3 and 13 is presented in Figure 18. These runs have the same value of suspended sediment volume fraction at the inflow. However, all the remaining controlling parameters are different (Table 2). As it can be seen, the temporal and spatial evolution of the generated currents is quite different. At the same times, the turbidity current in Run 3 has travelled longer distances $(5 \mathrm{~m}$ in $10 \mathrm{sec})$ than the turbidity current in Run $13(3.5 \mathrm{~m}$ in 10 sec. Moreover, the formation of the internal hydraulic jump, after the entrance of the current to the expansion table, is more intense in the case of Run 3. In both cases a slight color change is observed at the iso-surfaces of sediment volume fraction at the expansion table, before the entrance of the turbidity current. The reason for this reduction in the sediment 
volume fraction values, at the top of the loose sediment layer, is that after the release of the current from the inflow gate, ambient water is pushed out of the computational domain from the outlet boundaries, for mass balance purposes. This outward ambient water movement at the open boundaries carries away some sediment from the top of the loose sediment layer, and therefore the volume fraction value at these regions of the computational domain is reduced.

Figure 19 shows vertical ZX-sections of suspended sediment volume fraction contours at $\mathrm{Y}=0 \mathrm{~m}$, for Runs 1 and 4, at fixed times $(\mathrm{t}=1,3,6$ and $9 \mathrm{sec})$. In these runs, only the initial suspended sediment volume fraction is different while all the other controlling parameters remain unchanged (Table 2). Up to $t=3 \mathrm{sec}$, the evolution of the generated flows are similar for both runs with the turbidity current of Run 1 having a slightly greater propagation speed. However, as the currents propagate further downstream, after time $\mathrm{t}=6 \mathrm{sec}$, the turbidity current generated in Run 4 travels at a lower speed $(2.3 \mathrm{~m}$ in $6 \mathrm{sec})$, compared with the turbidity current generated in Run 1 ( $2.6 \mathrm{~m}$ in $6 \mathrm{sec}$ ), with a weakened head. Moreover, in the turbidity current generated in Run 1 the dense and dilute concentration layers are more evident than in the case of Run 4.

Contours of the X-velocity component of the flow, for numerical Runs 8 and 9 are illustrated in Figure 20, in vertical $\mathrm{ZX}$-sections at $\mathrm{Y}=0 \mathrm{~m}$, for fixed times $(\mathrm{t}=2,4$ and $6 \mathrm{sec})$. These runs have the same values of suspended sediment diameter and channel slope while all the other controlling parameters are varied (Table 2). As it can be observed, the global structure of the generated currents is quite similar. However, the turbidity current generated in Run 9 travels at a slower speed $(2.4 \mathrm{~m}$ in $6 \mathrm{sec})$ than the turbidity current in Run $8(2.6 \mathrm{~m}$ in 6 sec), having a slightly smaller thickness.

Finally, in order to visualize the differences in the flow dynamics for all the numerical runs conducted, from a quantitative point of view, the flow front advance with respect to time, for each of these runs is depicted in Figure 21. As it can be observed, the flow front advance with respect to time is quite different even for runs with small differences in the initial conditions or for runs were only one of the controlling parameters is altered (Table 2).

All the above observations indicate that flow dynamics of turbidity currents are highly dependent on various important controlling parameters, such as the inflow velocity, the channel bed slope, the initial sediment concentration, the sediment grain size and the channel bed roughness. The combined influence of these parameters can dramatically alter important flow characteristics such as the velocity and concentration structure as well as the thickness of the generated turbidity currents.

In order to examine the corresponding sensitivity in the resulting changes within the 
thin sediment layer that covers the expansion table before the beginning of the simulations, Figure 22 illustrates the initial $(t=0 \mathrm{sec})$ and final $(\mathrm{t}=15 \mathrm{sec})$ states of sediment volume fraction contours (in a $\mathrm{ZX}$ plane at $\mathrm{Y}=0 \mathrm{~m}$ ), isolating the proposed layer (cell zone 1 in Figure 5), for the case of numerical Runs 3 and 14 that show the maximum differences in the flow front position with respect to time curves (Figure 21). As it can be seen in both cases, the passage of the turbidity current after its entrance to the expansion tank causes considerable erosion to the loose sediment layer that initially covers the expansion table. At flow time $\mathrm{t}=0$ $\mathrm{sec}$, the sediment layer has a uniform sediment volume fraction value of 0.56 . At $t=15 \mathrm{sec}$ (end of numerical simulations), the downstream part of the layer has relatively high values of sediment volume fraction, while the upstream part has relatively low values. This denotes that the upstream part of the sediment layer has been eroded from the turbidity current passage, due to the entrainment of particles from the loose bed layer into the expanding turbidity current. However, the difference in the generated flow dynamics between these two runs (depicted in Figure 21) has a direct impact in the eroded regions shown in Figure 22. Therefore in the case of Run 14, where the generated turbidity current is less severe and travels with a much slower speed, the eroded region is limited at a small part, at the upstream end of the expansion tank (up to $\mathrm{X}=3.8 \mathrm{~m}$ ), while in the case of Run 3 the eroded region extends further downstream (up to $X=5.4 \mathrm{~m}$ ). Therefore, it can be concluded that the controlling flow parameters considered in the loose bed runs are equally important both for the flow dynamics of the generated currents (flow velocity, concentration and current thickness) as well as for the degree of their interaction with the underlying loose bed layer.

\section{Conclusions}

In this paper a 3D numerical model, which simulates the dynamics and flow structure of turbidity currents through a multiphase approach is used. Unlike most of the previous numerical investigations that treat the turbidity current flow as a quasi single-phase approach where the transport of sediment particles is taken into account through an advection-diffusion equation for sediment concentration, the present numerical approach assumes that the sediment-laden flow consists of solid and fluid phases, which are separate phases, treated as interpenetrating continua. The space occupied by each phase is represented by the volume fraction $\alpha(0<\alpha<1)$ and the laws for the conservation of mass and momentum are satisfied by each phase individually and coupling is achieved through pressure and interphase exchange coefficients. 
The proposed numerical approach is tested and verified through detailed qualitative and quantitative comparison with experimental data. The comparison of the overall numerical and experimental results indicates that the proposed numerical approach, can generally give fairly realistic predictions regarding the global flow structure of turbidity currents and it can also capture various important flow aspects such as the effect of particle deposition rates on their spatial and temporal evolution, the formation of distinct velocity and concentration layers in the body of the currents, the formation of internal hydraulic jumps, the mixing induced instabilities at their interface with the ambient water as well as their interaction with underlying loose sediment beds. The main advantages of the proposed, multiphase numerical approach, in relation to previous quasi single-phase approaches are the following:

- A separate velocity field is calculated for each phase (water and sediment classes).

- The RNG $k-\varepsilon$ turbulence model is used for turbulence closure that significantly increases the applicability of the proposed numerical approach, as it can also account for turbidity current flows with low Reynolds numbers. Furthermore, with the use of the RNG $k-\varepsilon$ turbulence model the accuracy in the case of rapidly strained flows is significantly improved, the effect of swirl in turbulence is taken into account and the turbulent Prandtl numbers are no longer user defined constants as they are calculated though an analytical formula.

- The total number of phases that can be simulated is only limited by the available memory of the computational resources. Hence, the proposed numerical approach can be used for the simulation of polydisperse turbidity currents that contain many classes of suspended sediment particles, which are more close to natural turbidity current flows.

- The proposed multiphase approach can handle a wide range of particulate loading, and therefore is capable for the simulation of both dilute and dense turbidity current flows.

- Since, the proposed numerical approach is based on the finite volume method, it can be applied in situations with complex geometries, like in the case of turbidity currents that are formed at natural, water basin beds (sea, lakes, reservoirs), where morphological anomalies are usually present. 
The further analysis and comparison of various extreme cases, among the numerical runs conducted, indicate that the flow of turbidity currents is highly sensitive to the variation of various controlling flow parameters, such as the inflow velocity, the initial suspended sediment concentration and grain size as well as the bed slope and roughness of the receiving basin. It is found that the combined influence of these parameters can dramatically alter important flow characteristics of the generated currents such as the velocity and concentration structure, the propagation speed of the front, the flow thickness and the degree of interaction with underlying loose sediment beds.

Summarizing, the present paper verifies and evaluates the usefulness of an uncommon numerical approach as a possible and quite suitable tool for future investigation on the hydrodynamic behavior of turbidity currents, allowing a wide range of flow parameters to be determined and continuously monitored with a relatively high accuracy. Moreover, the overall results of the present paper add a significant contribution to the evaluation of the capacity of CFD-based numerical models for the simulation of complex flow phenomena and the understanding of the physics of three dimensional turbidity currents.

For future investigation the response and relative importance of the individual flow parameters of turbidity currents can be further assessed performing various wide series of numerical experiments where the controlling parameters that are clearly identified in the present paper are isolated in each case, in order to quantify their relative importance and their corresponding impact at the flow properties of such complex flows.

\section{Acknowledgements}

The authors would like to thank Dr. Charlotte Gladstone and Dr. Jaco H. Baas for making their experimental data available for comparison purposes, as well as all the reviewers for the useful comments and recommendations on the initially submitted manuscript. The authors would also like to acknowledge the financial support from the Beachmed-e European Research Project.

\section{References}

1. Mulder T, Alexander J (2001) The physical character of subaqueous sedimentary density flows and their deposits. Sedimentology 48: 269-299

2. Janbu NE, Nemec W, Kirman E, İzaksoy V (2007) Facies anatomy of a channelized sandrich turbiditic system: the Eocene Kusuri Formation in the Sinop Basin, north-central Turkey. In: Nichols, G., Paola, C. and Williams, E.A. (Eds.), Sedimentary Environments, Processes and Basins - A Tribute to Peter Friend. International Association of Sedimentologists Special Publication (in press) 
3. Posamentier HW, Kolla V (2003) Seismic geomorphology and stratigraphy of depositional elements in deep-water settings. Journal of Sedimentary Research 73 (3):367-388

4. Saller A, Werner K, Sugiaman F, Cebastiant A, May R, Glenn D, Barker C (2008) Characteristics of Pleistocene deep-water fan lobes and their application to an upper Miocene reservoir model, offshore East Kalimantan, Indonesia. AAPG Bulletin 92 (7):919-949

5. Lovell JPB (1971) Control of slope on deposition from small-scale turbidity currents : experimental results and possible geological significance. Sedinientology 17:81-88

6. Simpson JE, Britter RE (1979) The dynamics of the head of a gravity current advancing over a horizontal surface. Journal of Fluid Mechanics 94:477-495

7. Britter RE, Linden PF (1980) The motion of the front of a gravity current travelling down an incline. Journal of Fluid Mechanics 99:531-543

8. Garcia M, Parker G (1989) Experiments on hydraulic jumps in turbidity currents near a canyon-fan transition. Science. DOI: 10.1126/science.245.4916.393.

9. Kneller BC, Bennett SJ, McCaffrey WD (1997) Velocity and turbulence structure of density currents and internal solitary waves: potential sediment transport and the formation of wave ripples in deep water. Sedimentary Geology 112:235-250

10. Gladstone C, Phillips JC, Sparks RSJ (1998) Experiments on bidisperse, constant-volume gravity currents: propagation and sediment deposition. Sedimentology 45: 833-843

11. Baas JH, Van Kesteren W and Postma G (2004) Deposits of depletive, quasi-steady high density turbidity currents: a flume analogue of bed geometry, structure and texture. Sedimentology 51:10531089

12. Garcia M (1994) Depositional turbidity currents laden with poorly sorted sediment. Journal of Hydraulic Engineering 120 (11): 1240-1263

13. Hartel C, Meiburg E, Necker F (2000) Analysis and direct numerical simulation of the flow at a gravitycurrent head: Part 1. Flow topology and front speed for slip and no-slip boundaries. Journal of Fluid Mechanics 418:189-212

14. Kassem A, Imran J (2001) Simulation of turbid underflows generated by the plunging of a river. Geology 29 (7):655-658

15. Heimsund S, Hansen EWM, Nemec W (2002) Computational 3D fluid-dynamics model for sediment transport, erosion and deposition by turbidity currents. In: Knoper, M. and Cairncross, B. (Ed.) Abstracts, International Association of Sedimentologists 16th International Sedimentological Congress, Rand Afrikaans Univ., Johannesburg, p. 151-152

16. Lavelli A, Boillat JL, De Cesare G (2002) Numerical 3D modelling of the vertical mass exchange induced by turbidity currents in Lake Lugano (Switzerland). Proceedings 5th International Conference on Hydro-Science and -Engineering (ICHE-2002), Reference: LCH-CONF-2002-012 Note: [355]

17. Necker F, Hartel C, Kleiser L, Meinburg E (2002) High-resolution simulations of particle driven gravity currents. International Journal of Multiphase Flow 28: 279-300

18. Cantero M, Garcia M, Buscaglia G, Bombardelli F, Dari E (2003) Multidimensional CFD simulation of a discontinuous density current. Proceedings of the XXX IAHR International Congress, Thessaloniki, Greece, 2003

19. Bombardelli FA, Cantero M I, Buscaglia GC, García MH (2004) Comparative study of convergence of CFD comercial codes when simulating dense underflows. Mec'anica Computacional Vol. XXIII G.Buscaglia, E.Dari, O.Zamonsky (Eds.) Bariloche, Argentina, November 2004

20. Imran J, Kassem A, Khan SM, (2004) Three-dimensional modeling of density current. I. Flow in straight confined and unconfined channels. Journal of Hydraulic Research 42(6):578-590

21. Blanchette F, Strauss M, Meiburg E, Kneller B, Glinsky ME (2005) High-resolution numerical simulations of resuspending gravity currents: Conditions for self-sustainment. Journal of Geophysical Research, 110, C12022, (doi: 10.1029/2005JC002927)

22. Huang H, Imran J, Pirmez C (2005) Numerical model of turbidity currents with a deforming bottom boundary. Journal of Hydraulic Engineering 131(4):283-293

23. Ooi SK, Constantinescu G, Weber LJ (2007) 2D Large-Eddy Simulation of Lock-Exchange Gravity Current Flows at High Grashof Numbers. Journal of Hydraulic Engineering 133 (9): 1037-1047.

24. Mehdizadeh A, Firoozabadi B, Farhanieh B, (2008) Numerical Simulation of Turbidity Current Using $\overline{v^{2}}-f$ Turbulence Model. Journal of Applied Fluid Mechanics 1 (2):45-55

25. Cantero MI, Balachandar S, Garcia MH (2008) An Eulerian-Eulerian model for gravity currents driven by inertial paricles. International Journal of Multiphase Flow 34:484-501

26. Cantero MI, Balachandar S, Garcia MH, Bock D (2008) Turbulent structures in planar gravity currents and their influence on the flow dynamics. Journal of Geophysical Research - Oceans, 113, C08018, (doi: 10.1029/2007JC004645) 
27. Singh J (2008) Simulation of suspension gravity currents with different initial aspect ratio and layout of turbidity fence. Applied Mathematical Modelling 32:2329-2346.

28. ANSYS FLUENT 6.3.26 User's Guide (2008)

29. Hinze JO (1975). Turbulence. Mc Graw-Hill Publishing Co., New York

30. Bombardelli FA, Jha SK (2009) Hierarchical modeling of the dilute transport of suspended sediment in open channels. Environmental Fluid Mechanics 9:207-235

31. Dey S (1999) Sediment threshold. Applied Mathematical Modelling 23:399-417

32. Farrell GJ, Stefan HG (1986) Buoyancy induced plunging flows into reservoirs and coastal regions. Tech.Rep. No.241, At. Anthony Falls Hydr. Lab., Univ. of Minnesota, Minneapolis

33. Cebeci T, Bradshaw P (1977) Momentum transfer in boundary layers. Hemisphere Publishing Corporation, New York

\section{Figure Captions}

Figure 1 Geometry of the physical problem which is simulated experimentally by Gladstone et al. [15]

Figure 2 Geometry, mesh and boundary conditions used in the numerical simulations reproducing the experiments of Gladstone et al. [15]

Figure 3 Grid dependency study in the case of numerical Run A, from the numerical simulations reproducing the experimental runs of Gladstone et al. [15]. The flow front advance with respect to time is depicted for three different meshes

Figure 4 Geometry of the physical problem which is simulated experimentally by Baas et al. [28]

Figure 5 Geometry, mesh and boundary conditions used in the numerical simulations reproducing the experiments of Baas et al. [16]

Figure 6 Grid dependency study in the case of numerical Run 1, from the numerical simulations reproducing the experiments of Baas et al. [16]. The flow front advance with respect to time is depicted for three different meshes

Figure 7 Comparison of numerical (present study) and experimental [15] results, of flow front advance with respect to time

Figure 8 Comparison of numerical (present study) and experimental [15] results, of distance reached by the flow fronts with respect to the initial percentage of coarse fraction (fixed times)

Figure 9 Comparison of numerical dimensionless velocity profiles (present study) with analogous experimental data [17], for numerical Runs A, D and $G$ that reproduce the experiments of Gladstone et al. [15]

Figure 10 Contours of velocity magnitude for time $t=5 \mathrm{sec}$, in a $\mathrm{ZX}$ section at $\mathrm{Y}=0 \mathrm{~m}$ (Numerical Run 1)

Figure 11 Fine sand volume fraction contours in a $\mathrm{Z}-\mathrm{X}$ plane at $\mathrm{Y}=0 \mathrm{~m}$, for flow time $\mathrm{t}=$ $7 \mathrm{sec}$ (Numerical Run 1)

Figure 12 Initiation and time evolution of internal hydraulic jump (Numerical Run 1).

Figure $13 \quad 3 \mathrm{D}$ iso-surfaces of fine sand volume fraction, for flow time $\mathrm{t}=15 \mathrm{sec}$ (Numerical Run 1)

Figure 14 (a) Vertical velocity profiles at $\mathrm{X}=2.5 \mathrm{~m}, \mathrm{Y}=0 \mathrm{~m}$, and $\mathrm{t}=15 \mathrm{sec}$, used for the calculation of the numerically simulated turbidity current heights, applying Equation 13 (Numerical Runs 1, 3 and 4), (b) Vertical volumetric concentration profiles at $\mathrm{X}=2.5 \mathrm{~m}, \mathrm{Y}=0 \mathrm{~m}$, and $\mathrm{t}=15 \mathrm{sec}$, used for the graphical estimation of the numerically simulated, dense layer thicknesses (Numerical Runs 1,3 and 4)

Figure 15 Variation of head velocity with respect to the initial suspended sediment concentration for Turbidity currents laden with fine Sand, very fine sand and coarse silt. Comparison of numerical (present study) and experimental [16] 
Figure $16 \quad$ Comparison of numerical dimensionless velocity profiles (present study) with analogous experimental data [17], for numerical Runs 1, 7 and 14 that reproduce the experiments of Baas et al. [16]

Figure 17 Simulated time series of density contour and velocity vector plots for numerical Runs A (100\% fine) and G (100\% coarse) at 0, 5, 10 and $30 \mathrm{sec}$ after the release of the dilute mixtures (lock-gate cases)

Figure 18 3D iso-surfaces of sediment volume fraction, for flow times $t=5,10$ and 15 sec, for numerical Runs 3 and 13 (loose bed cases)

Figure 19 Sediment volume fraction contours in a $\mathrm{Z}-\mathrm{X}$ plane at $\mathrm{Y}=0 \mathrm{~m}$, for flow times $\mathrm{t}=$ $1,3,6$ and $9 \mathrm{sec}$, for numerical Runs 1 and 4 (loose bed cases)

Figure $20 \mathrm{X}$-velocity contours in a $\mathrm{Z}$-X plane at $\mathrm{Y}=0 \mathrm{~m}$, for flow times $\mathrm{t}=2,4$ and $6 \mathrm{sec}$, for numerical Runs 8 and 9 (loose bed cases)

Figure 21 Sensitivity of flow front advance with respect to time for numerical Runs 1, 3, 4, 7, 8, 9, 13 and 14 (loose bed cases), in relation to the variation of inflow velocity, initial suspended sediment concentration, suspended sediment grain size, channel bed slope and roughness (Table 3)

Figure 22 Initial and final state of sediment volume fraction for the thin layer of loose sediment that initially covers the expansion table (cell zone 1, Figure 5), shown at a ZX-plane at $\mathrm{Y}=0 \mathrm{~m}$, for numerical Runs 3 and 14 (loose bed cases).

\section{Table Captions}

Table 1 Relative initial proportions by mass, of each particle size grade used in the experimental runs of Gladstone et al. [15]

Table 2 Initial conditions of the loose-bed experimental [16] and numerical (present study) runs

Table 3 Comparison of numerical (present study) and experimental [16] values for mean height and dense layer thickness of turbidity currents (loose bed runs 1, 3 and 4) 


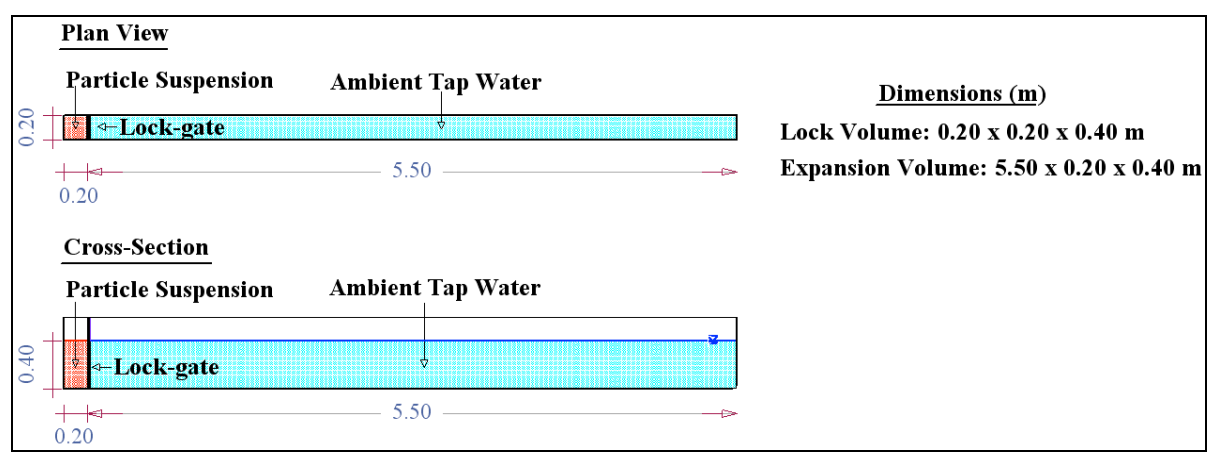

Fig. 1

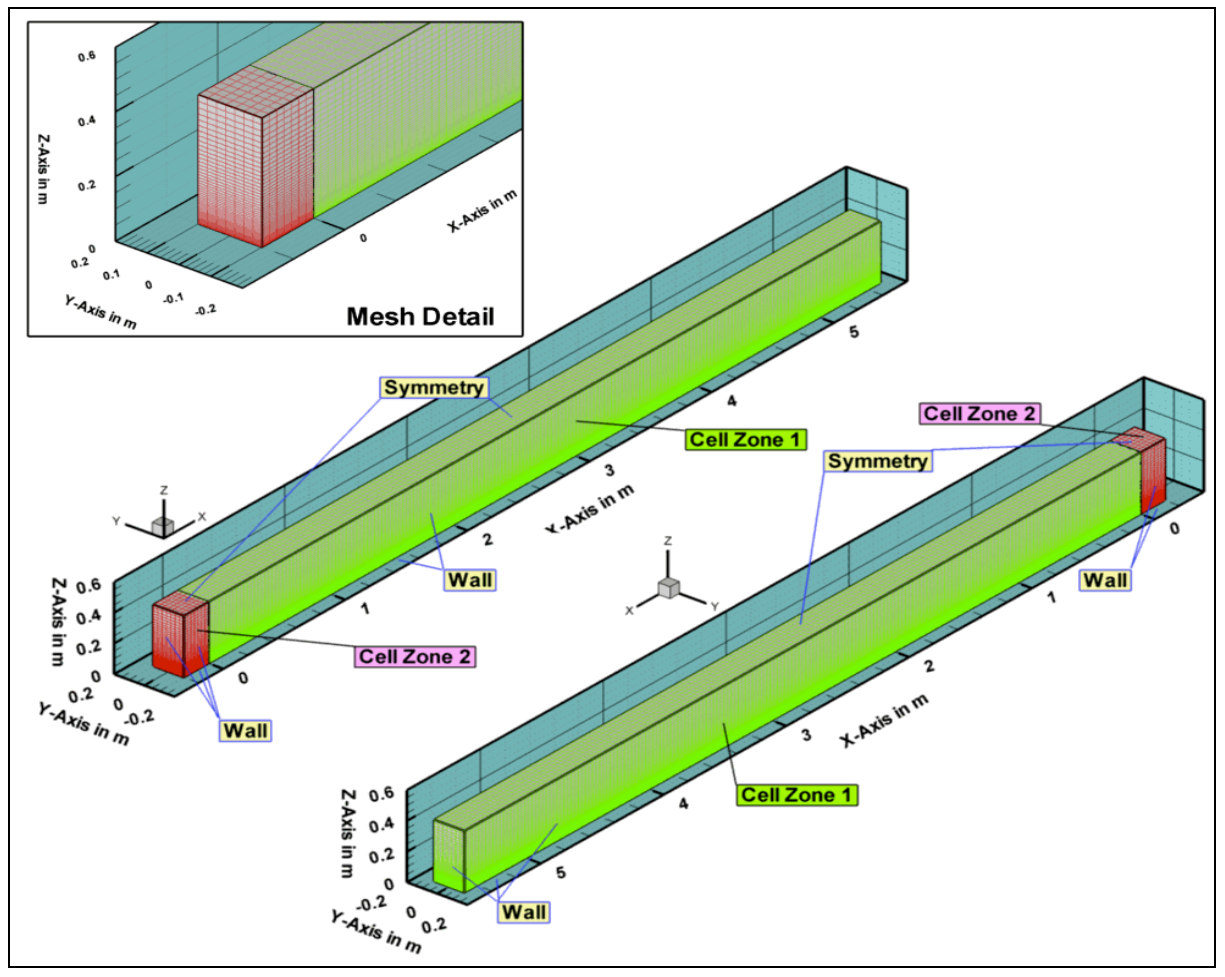

Fig. 2 


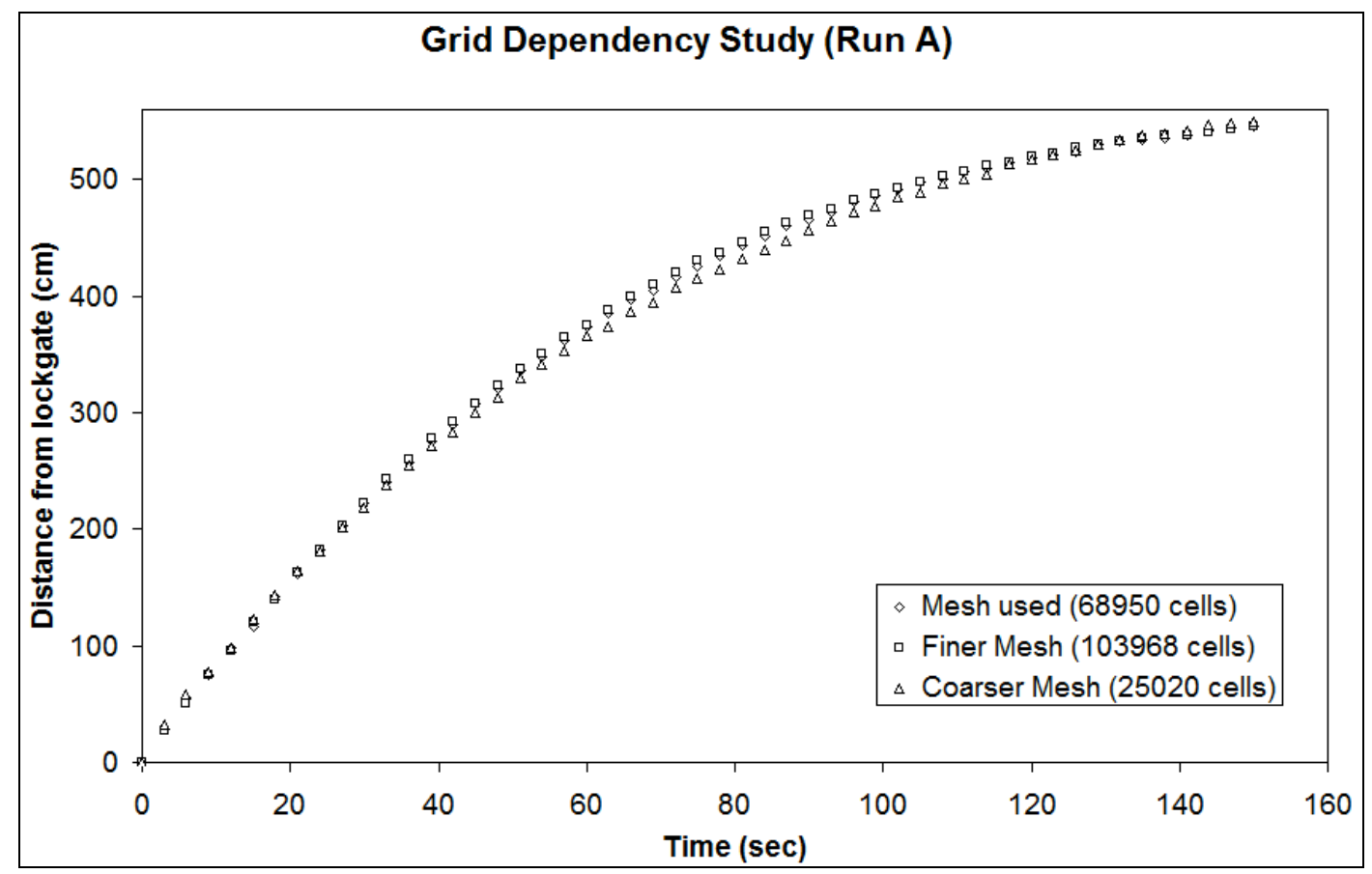

Fig. 3

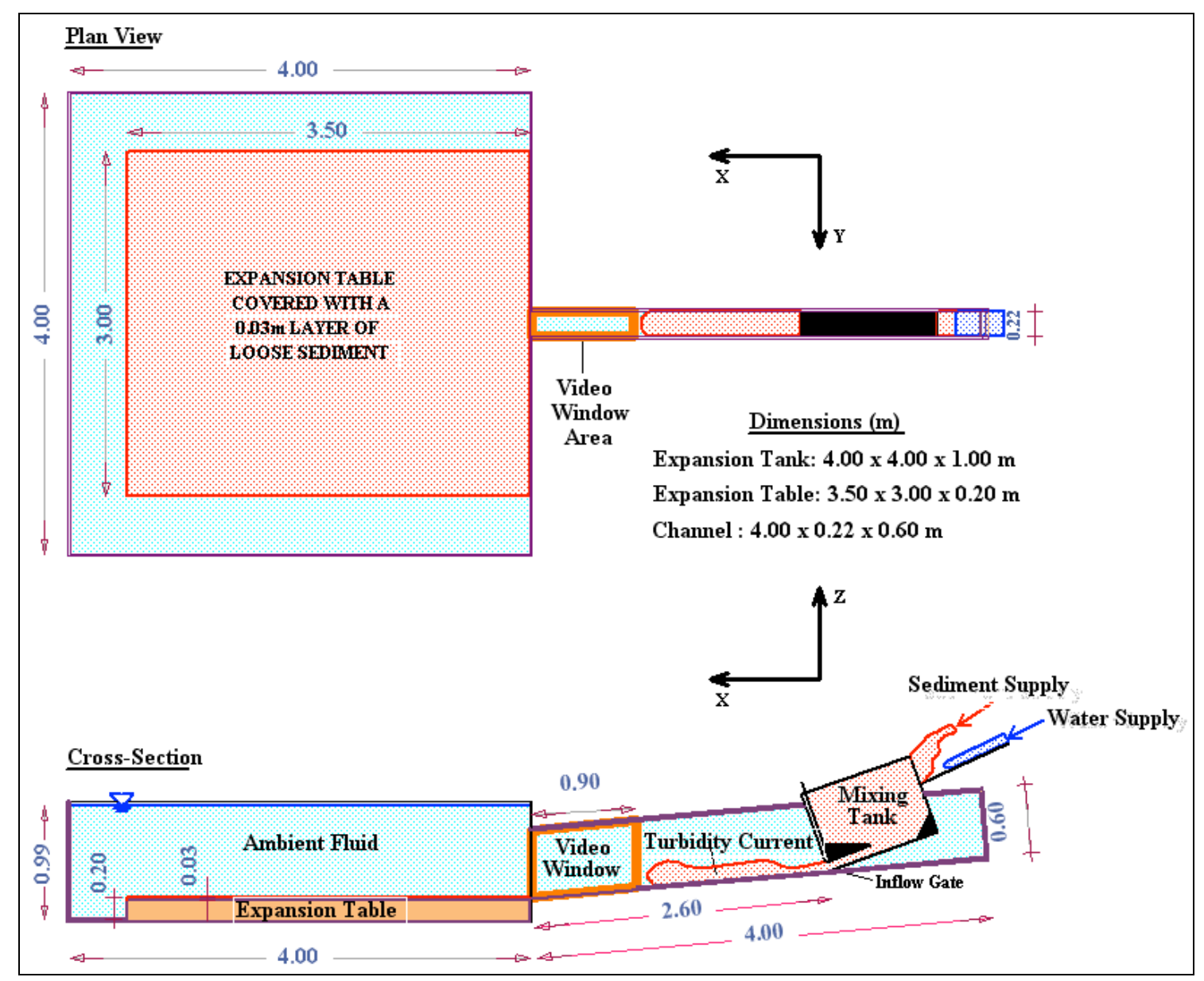

Fig. 4 


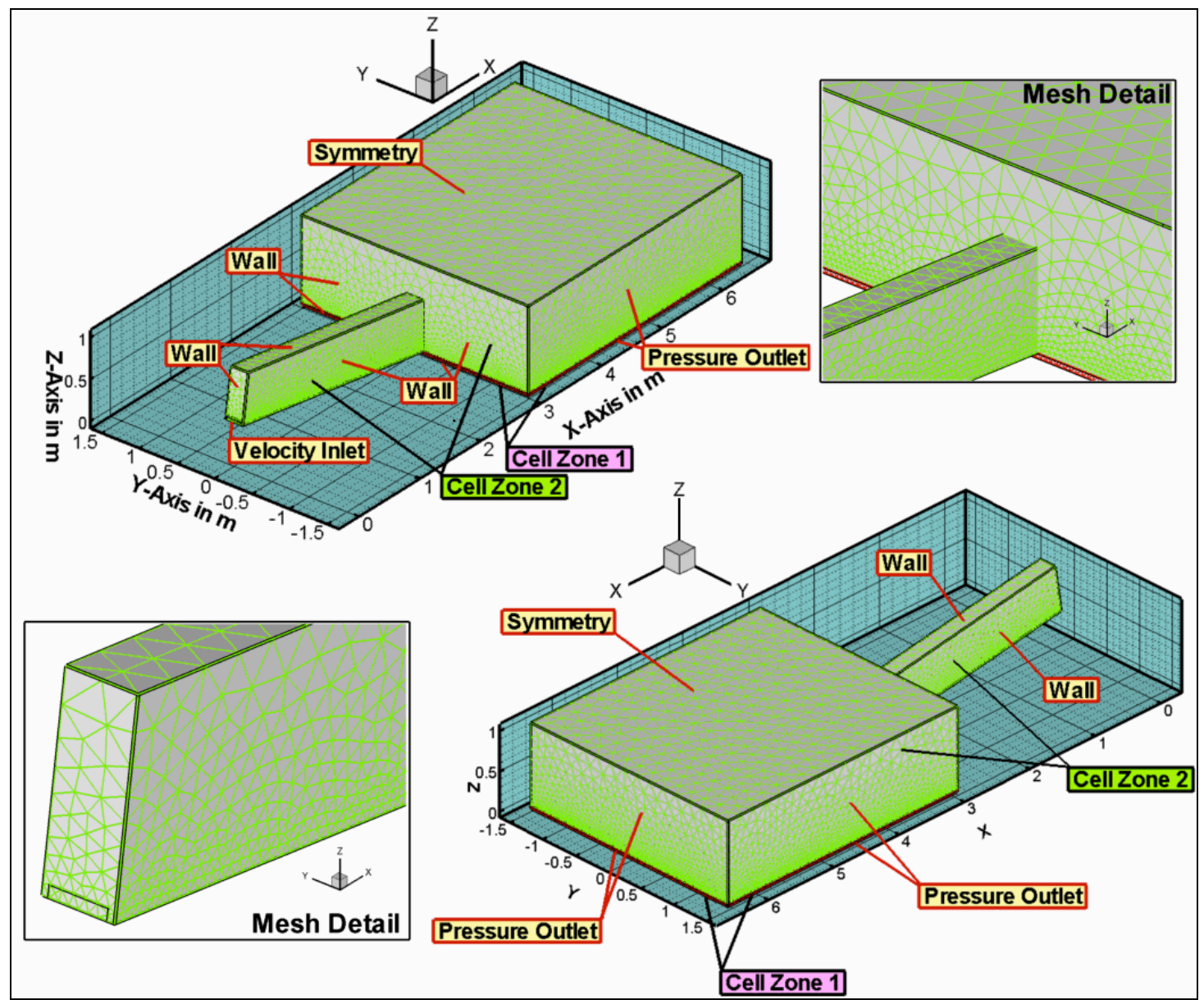

Fig. 5

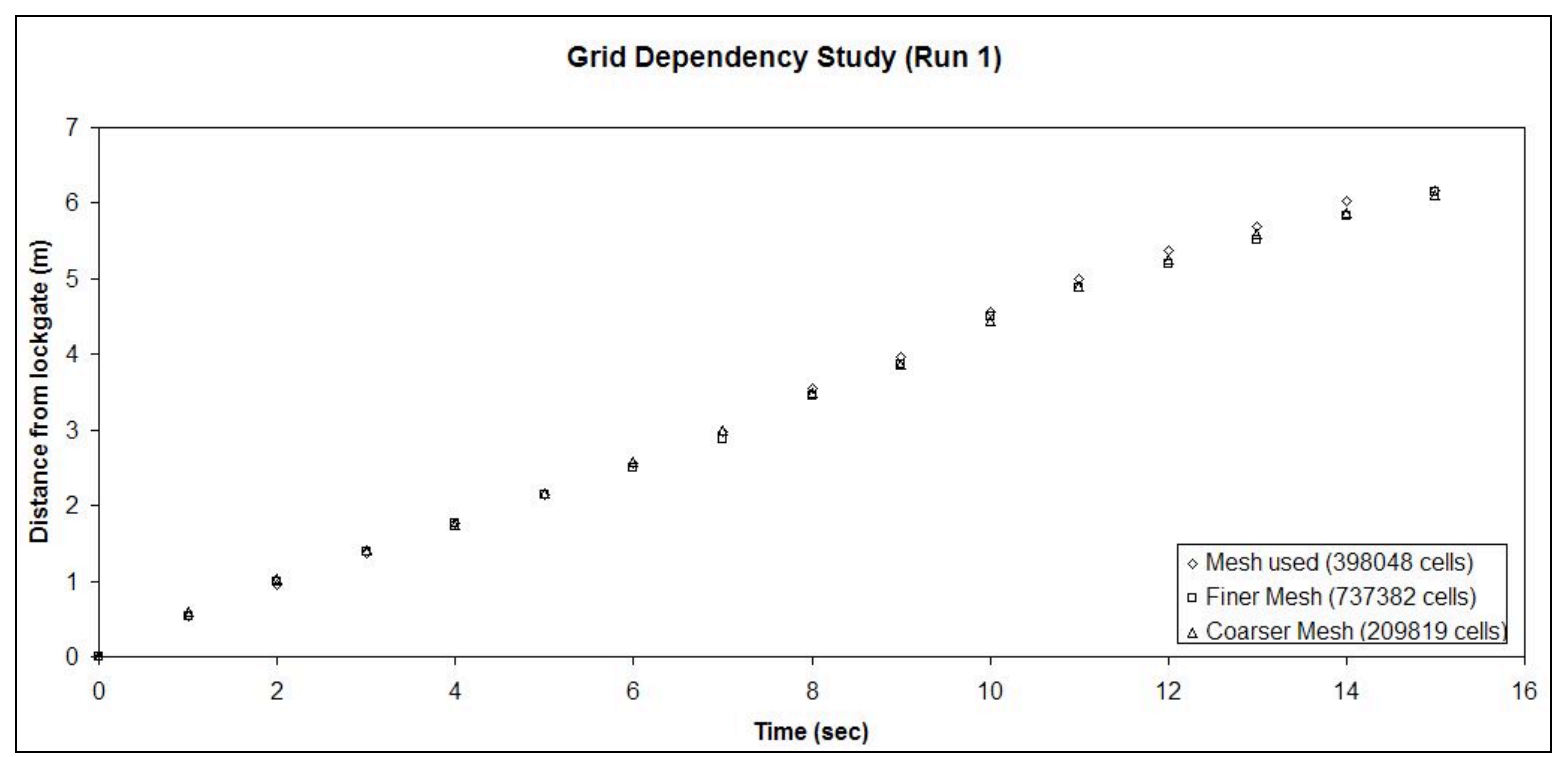

Fig. 6 


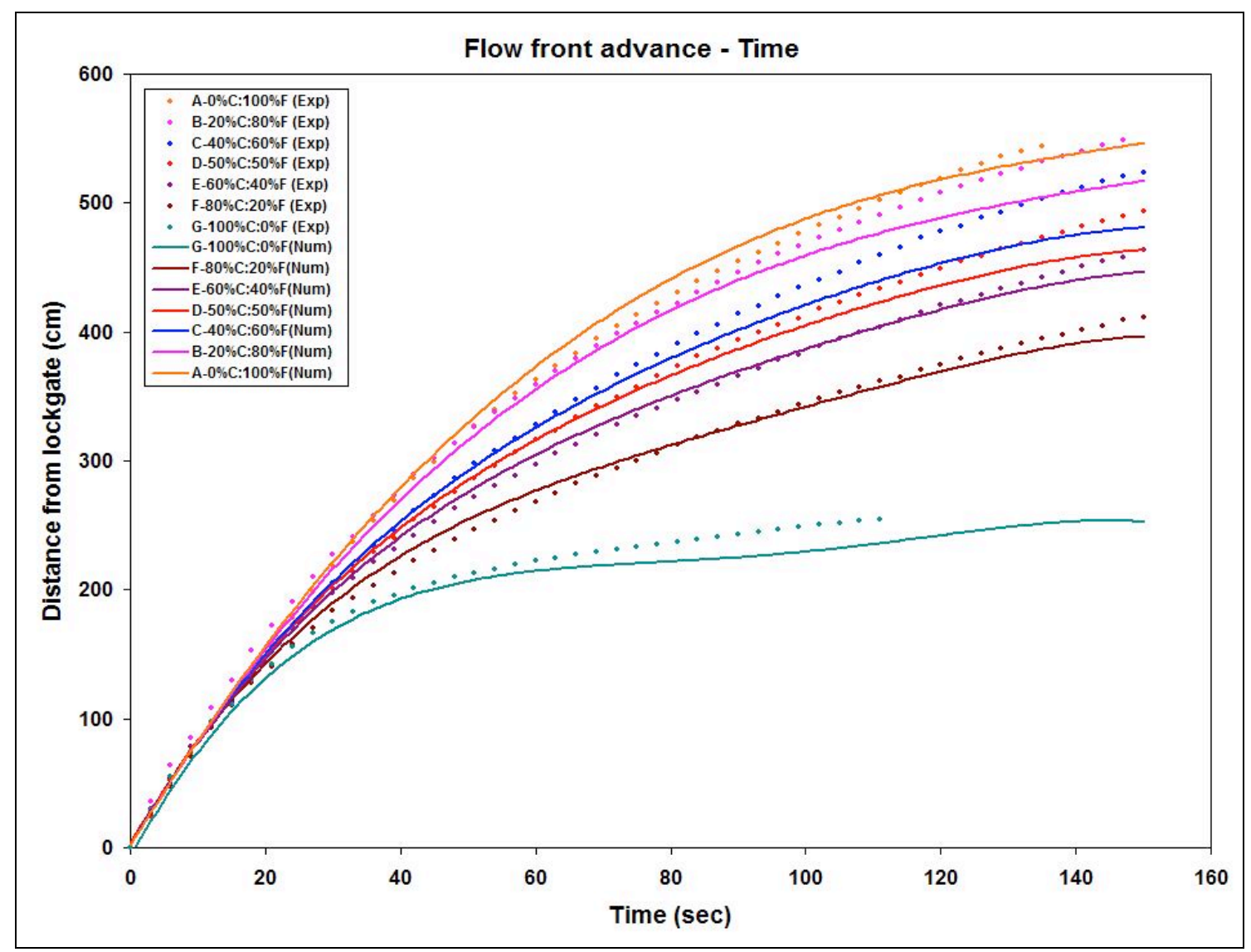

Fig.7

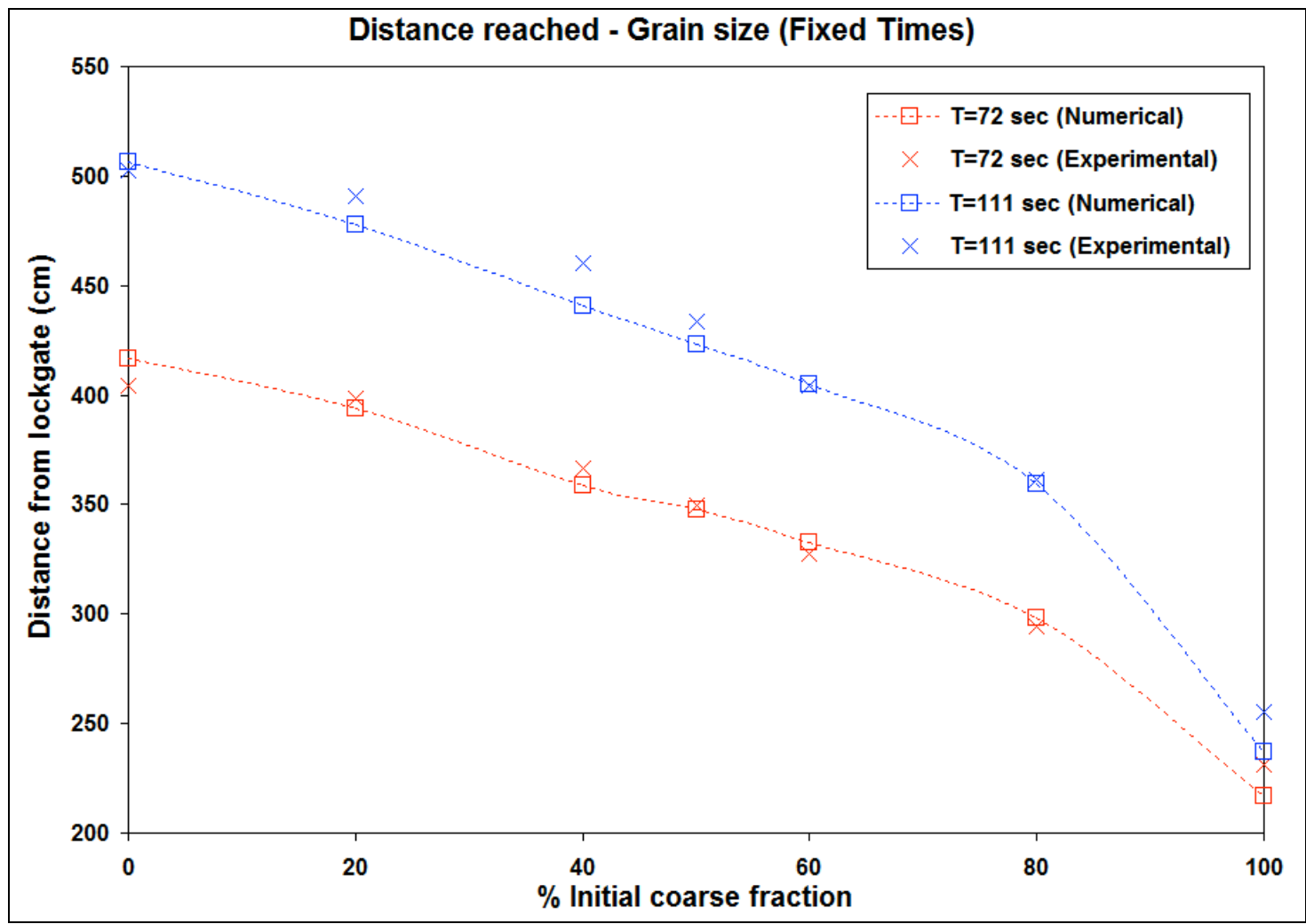

Fig. 8 


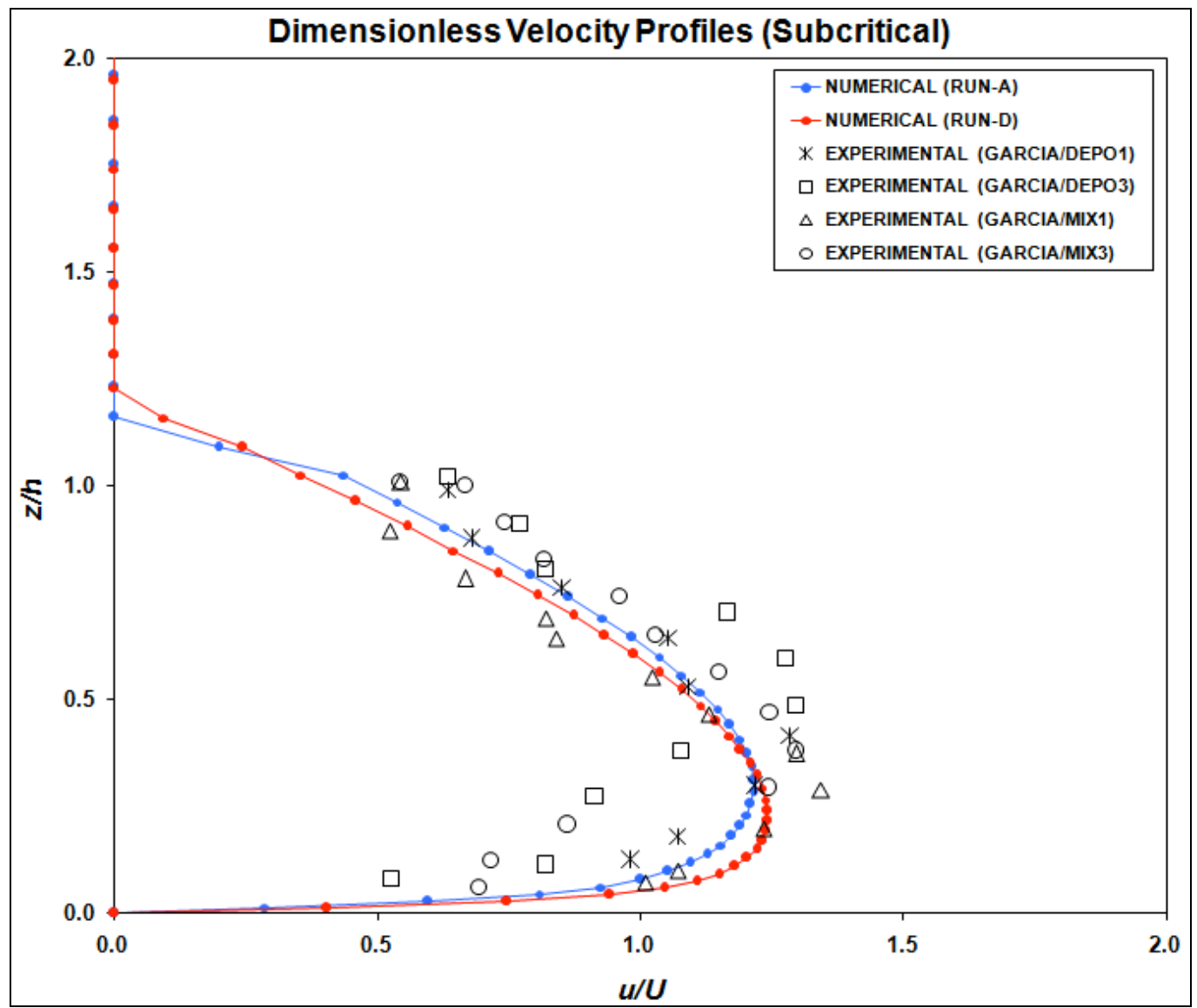

Fig. 9

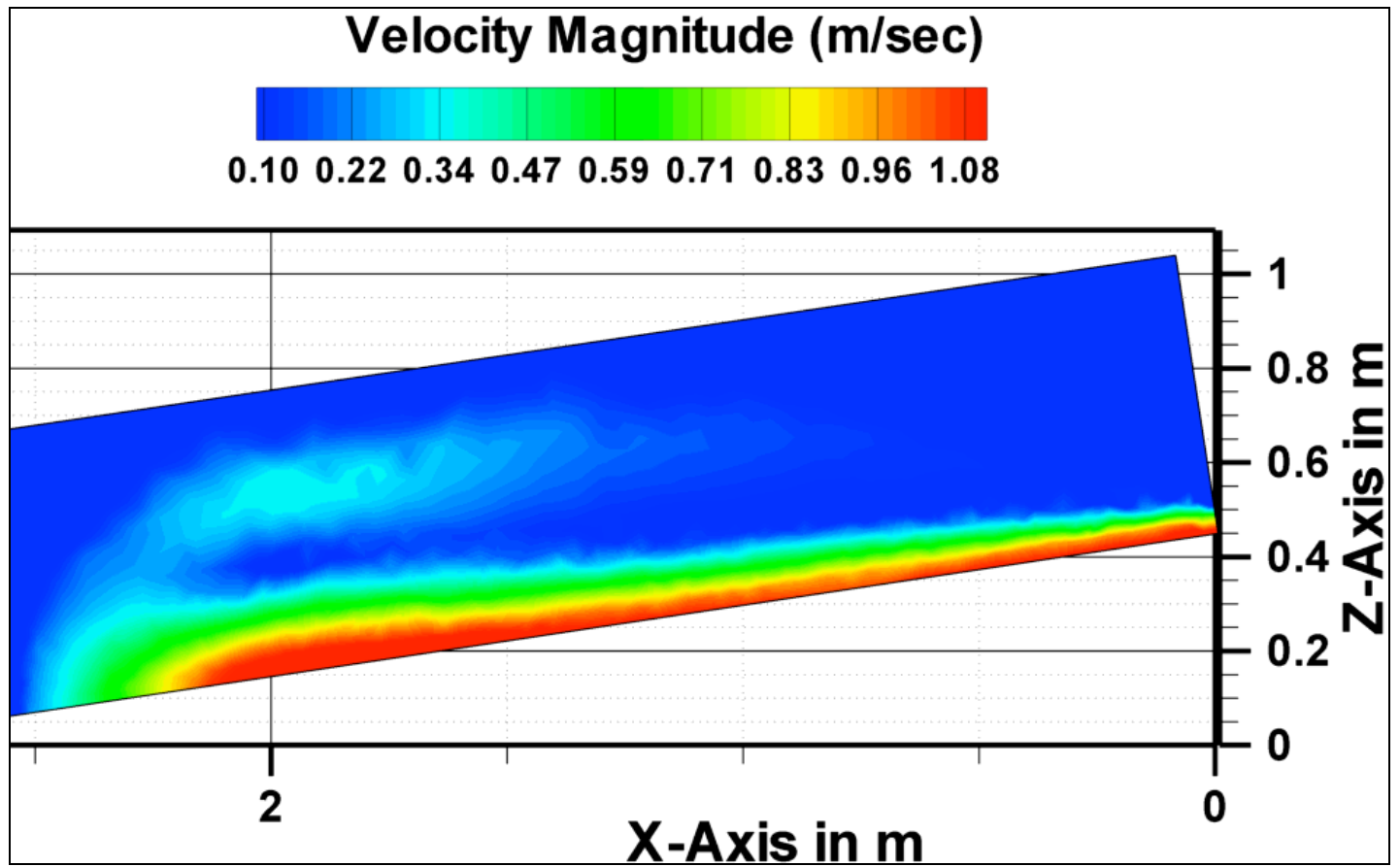

Fig.10 


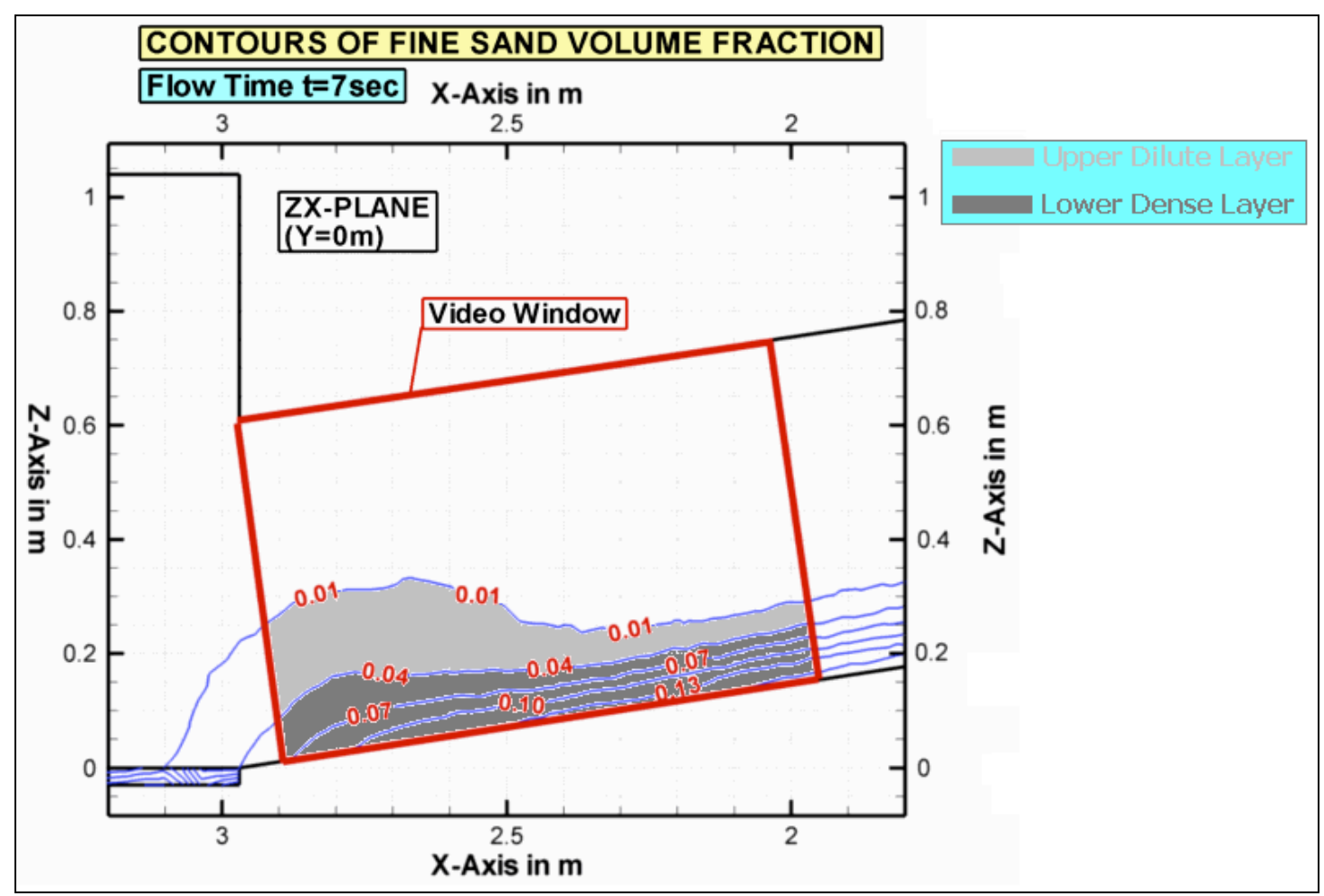

Fig. 11

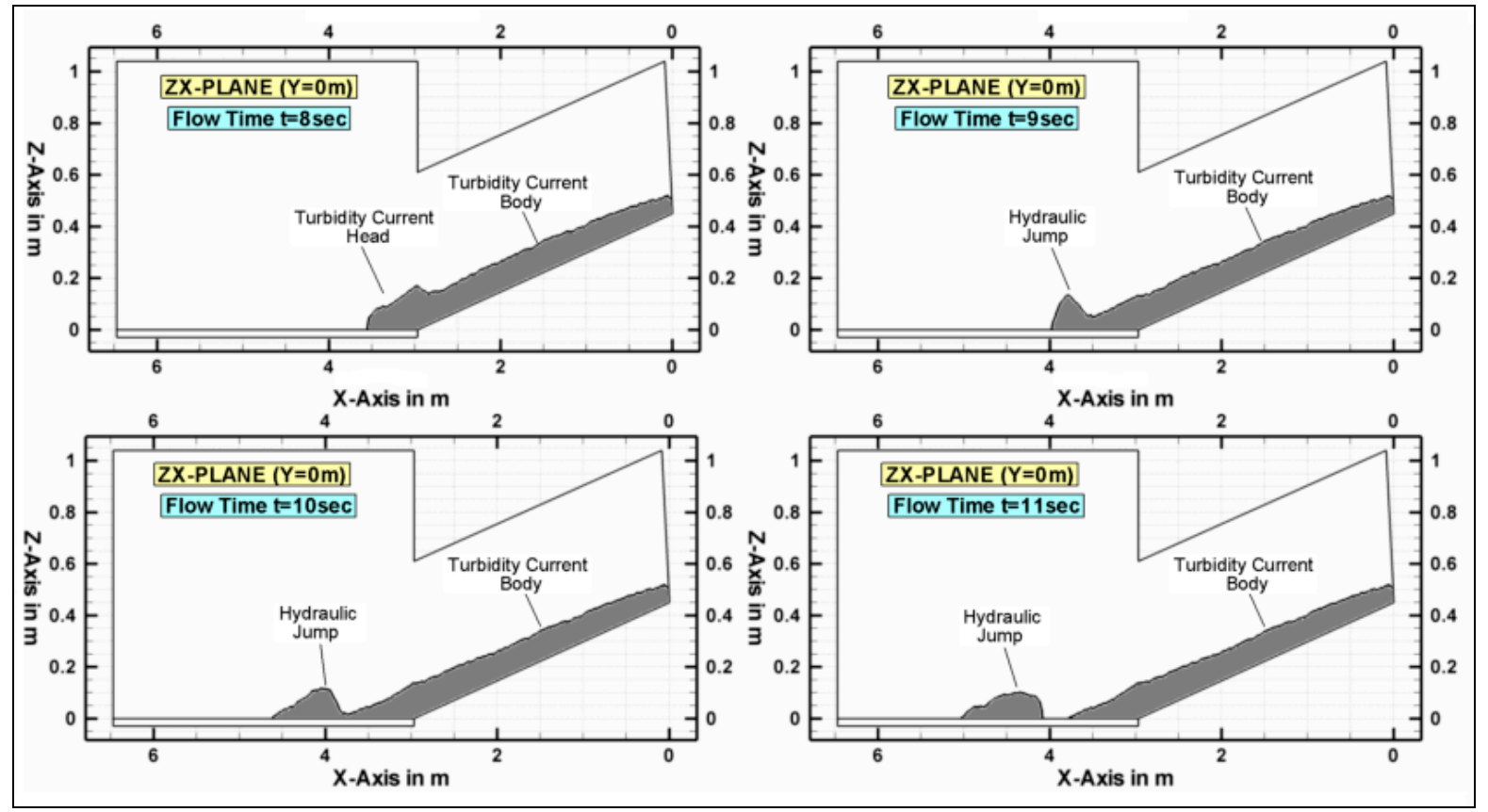

Fig. 12 


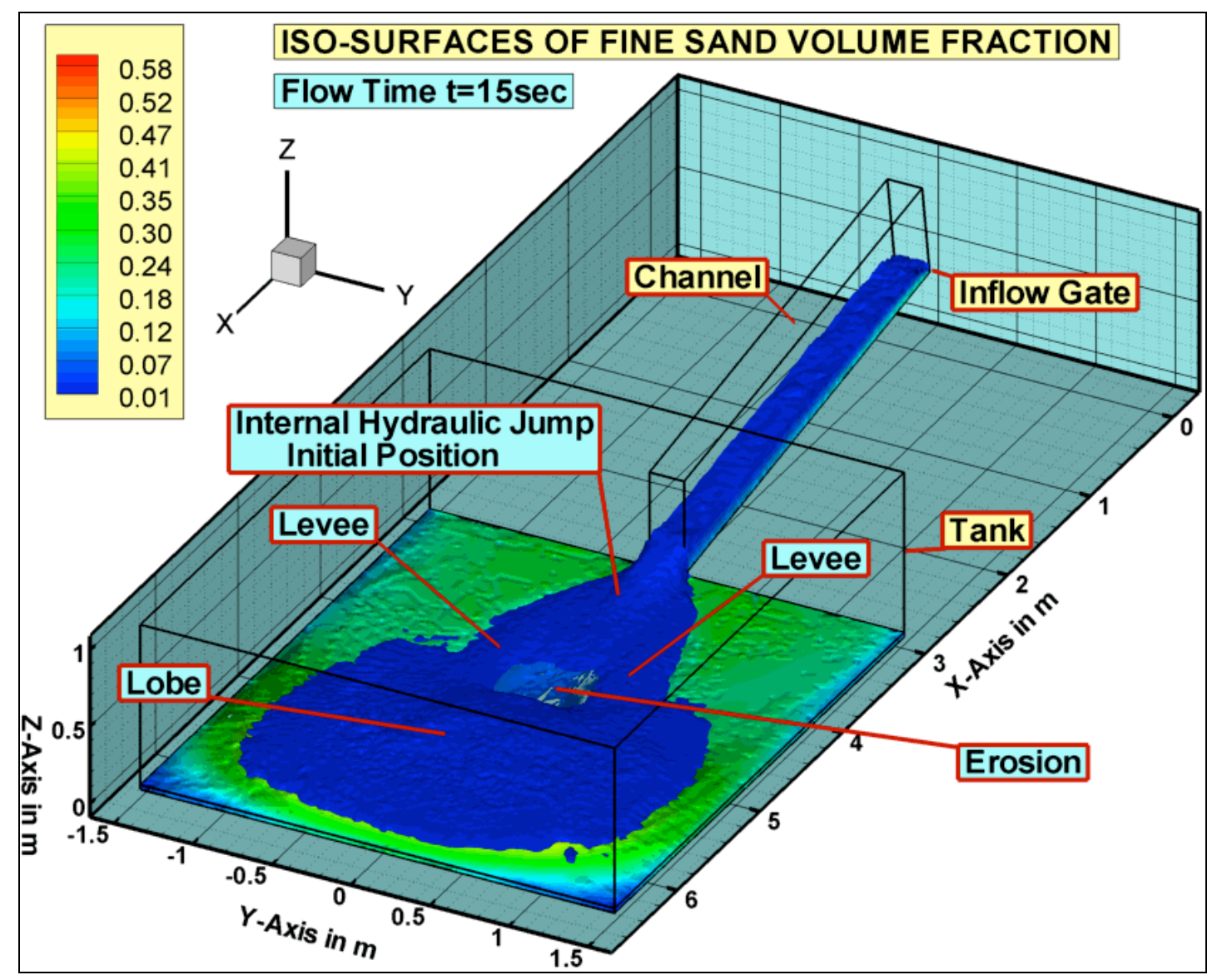

Fig. 13

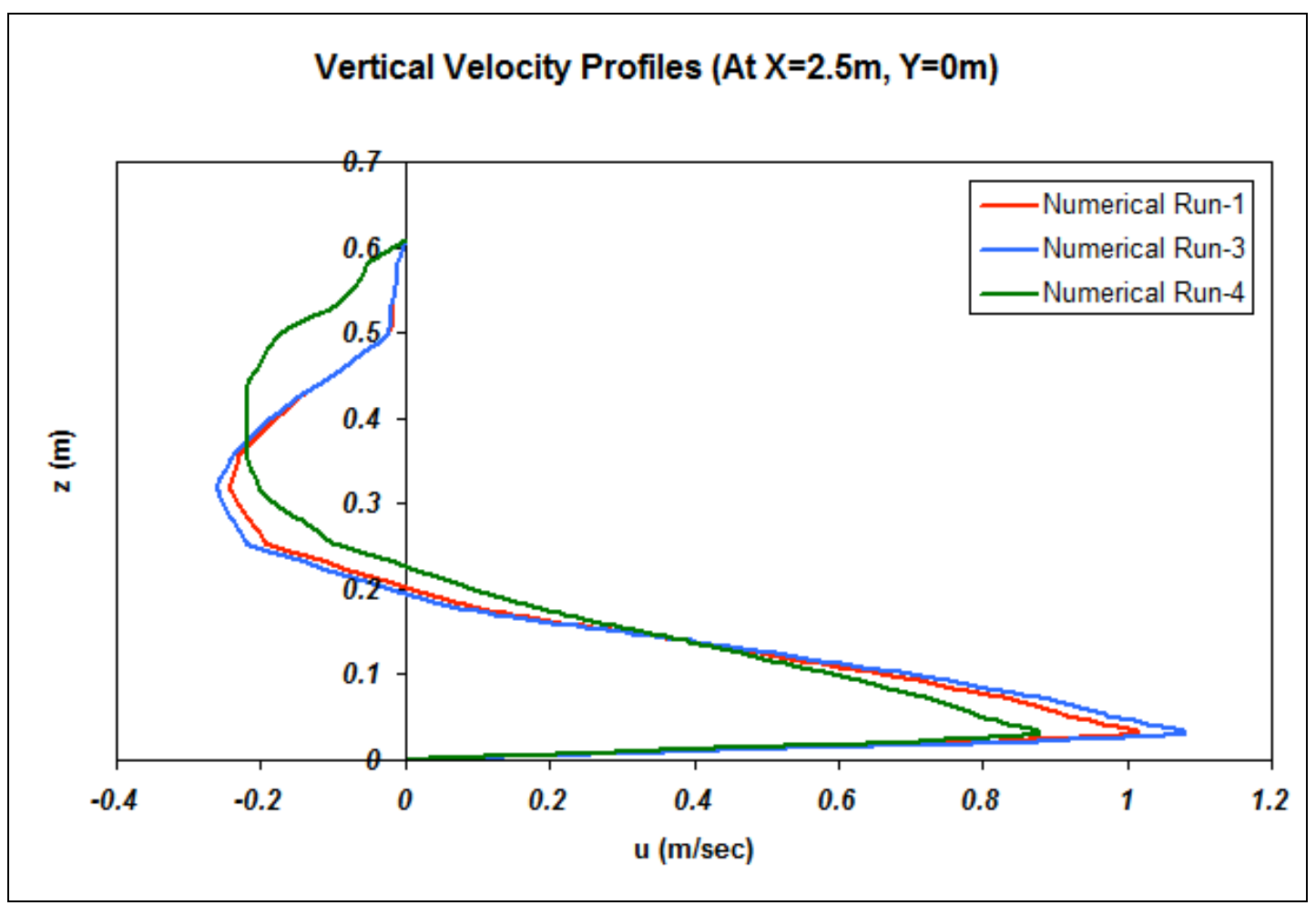

Fig. 14 (a) 


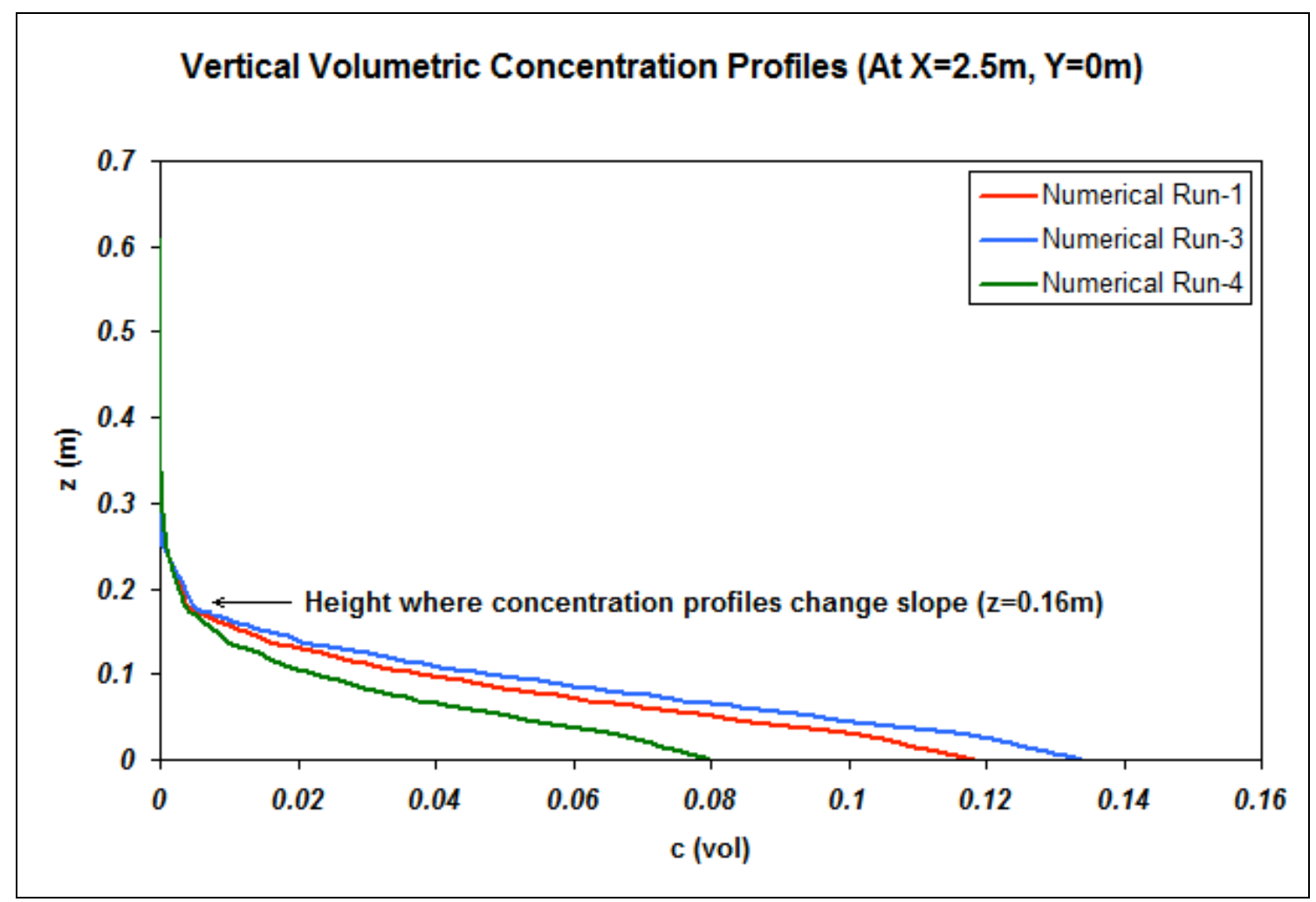

Fig. 14 (b)

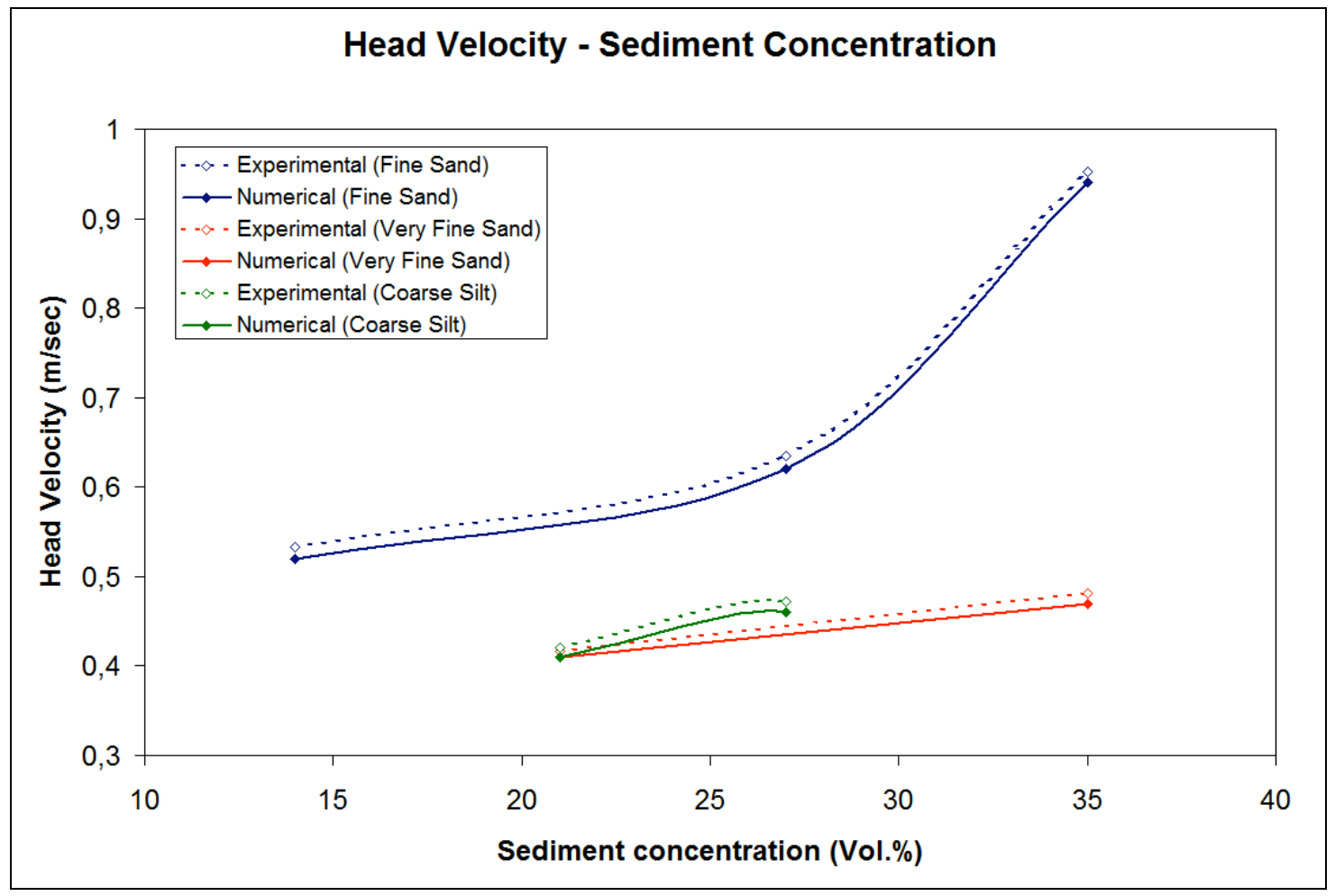

Fig. 15 


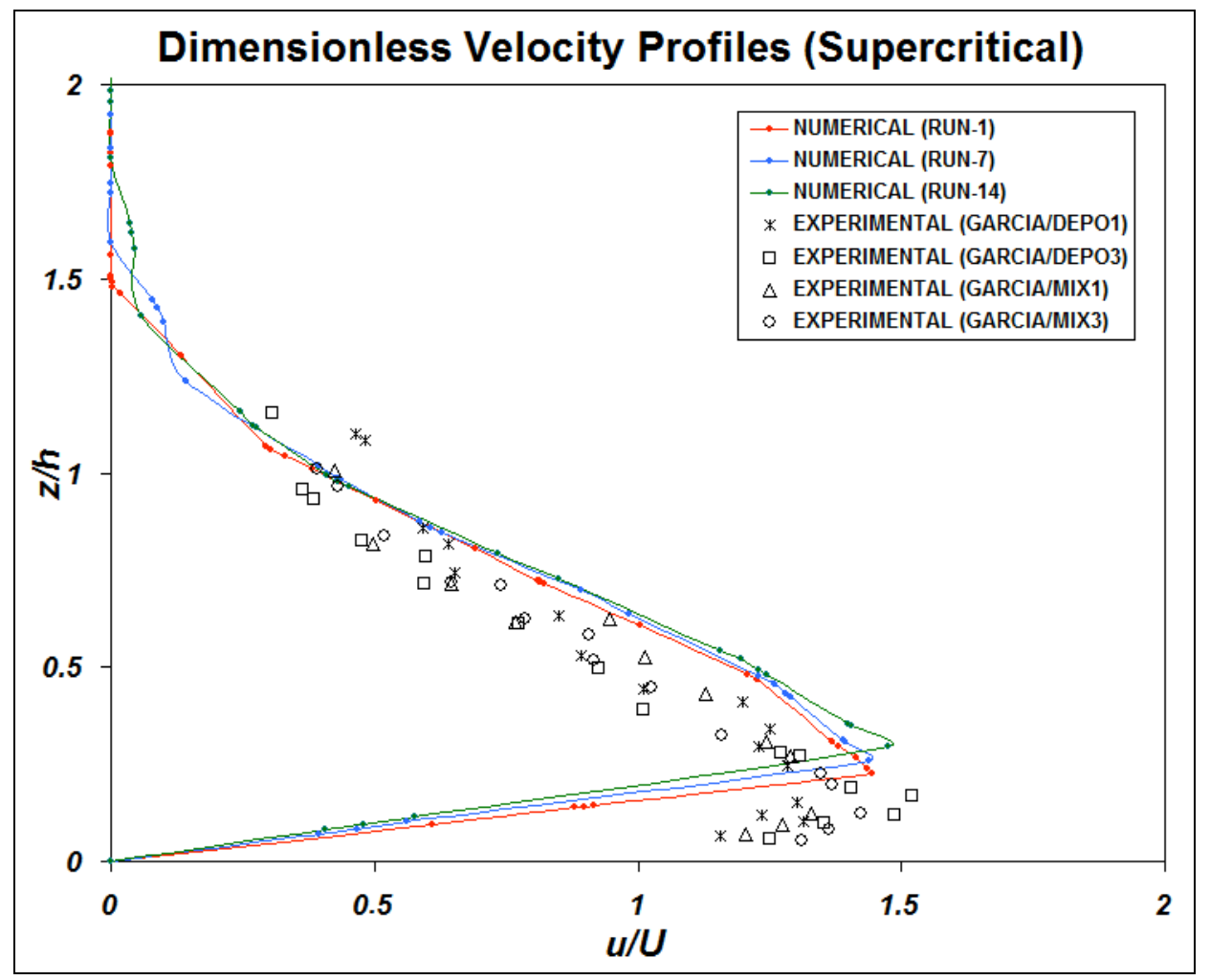

Fig. 16

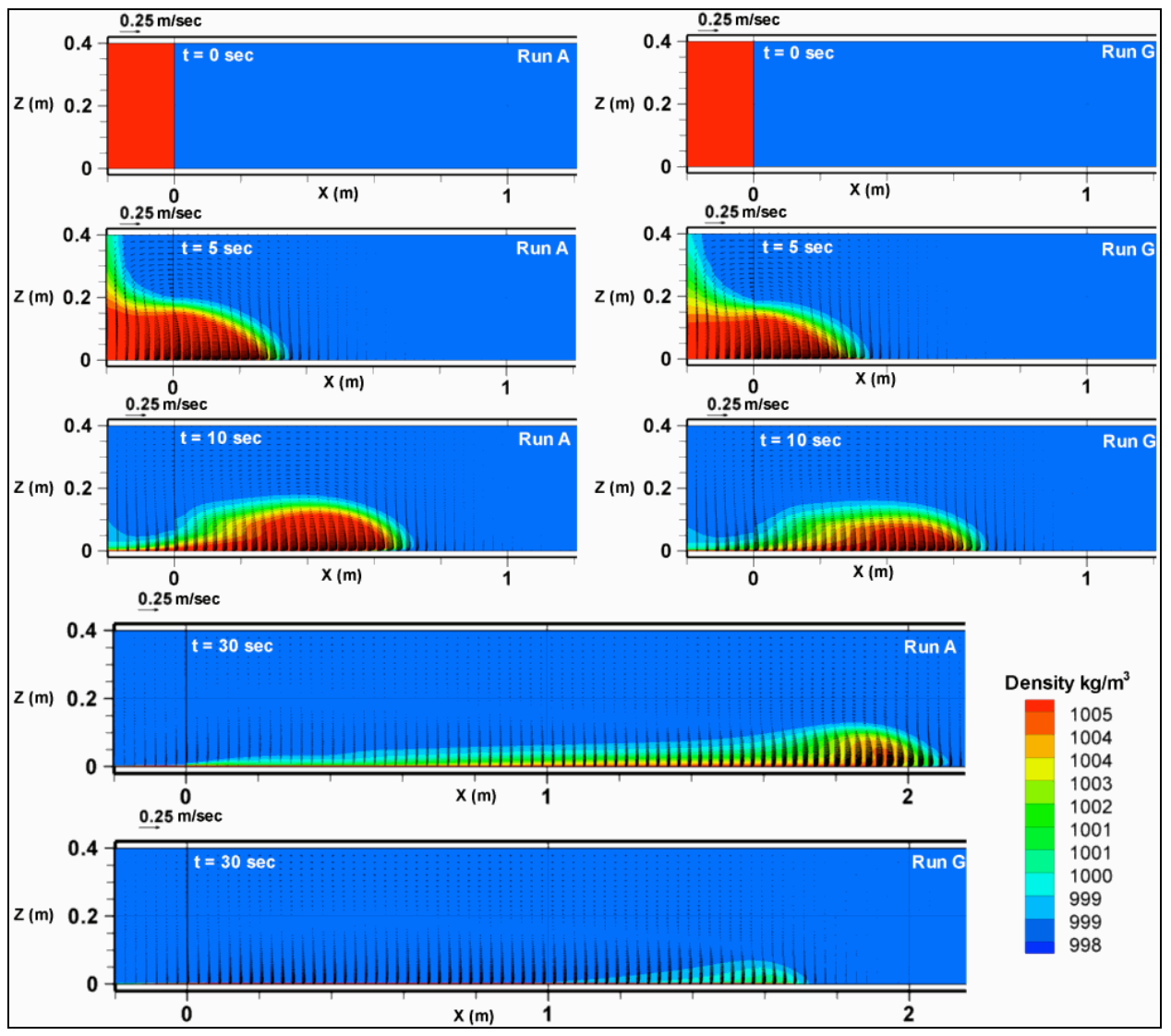

Fig. 17 


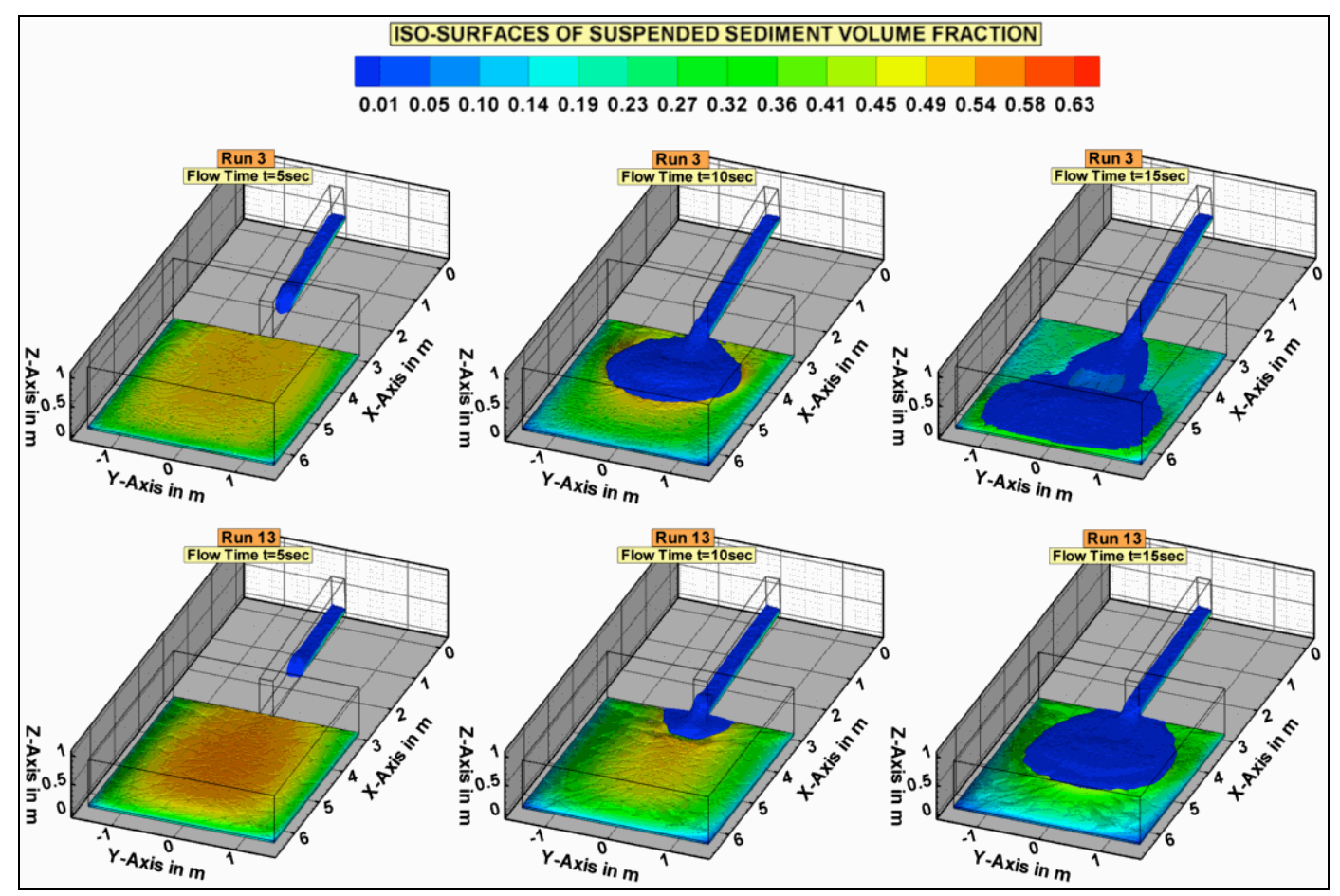

Fig. 18

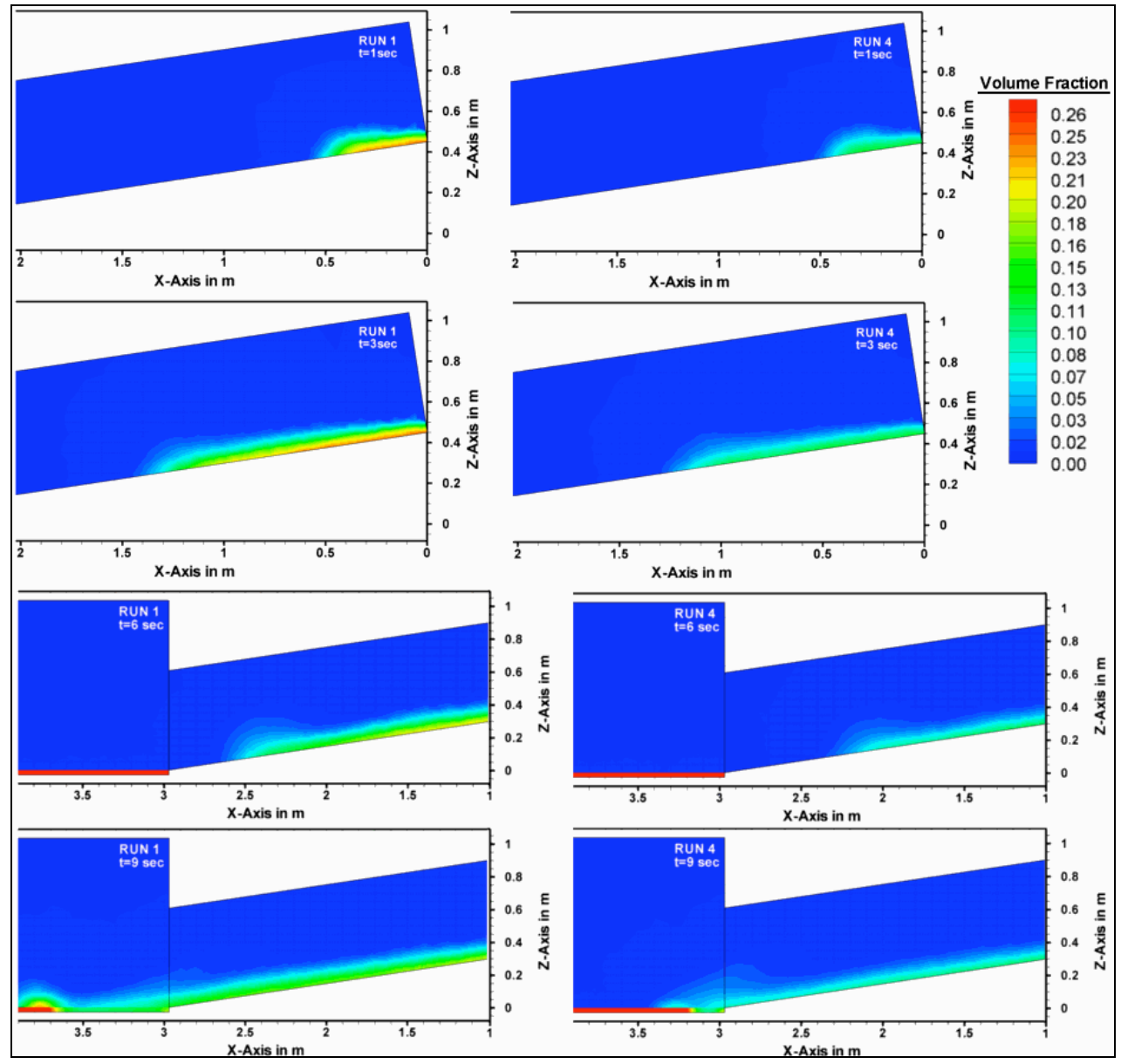

Fig. 19 


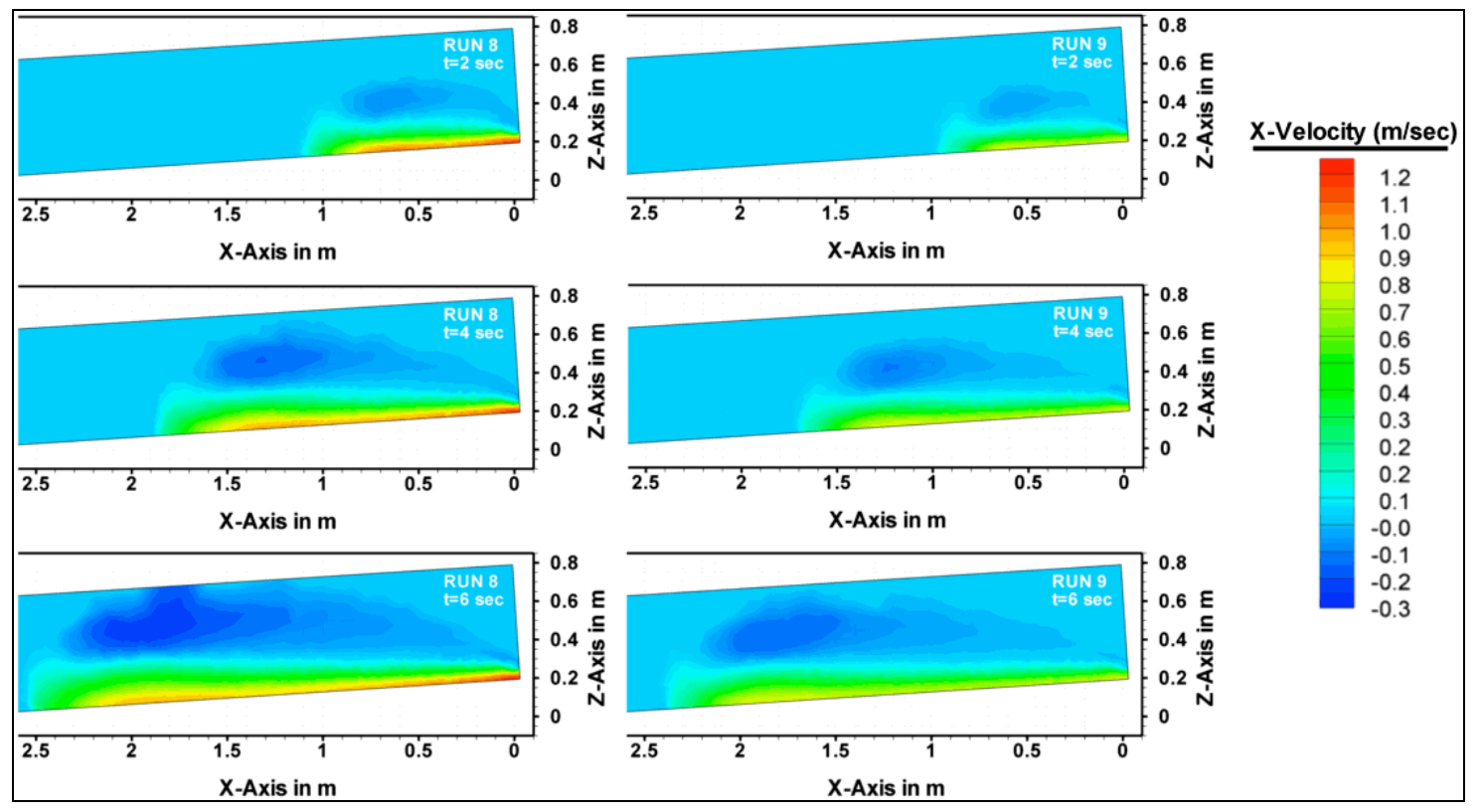

Fig. 20

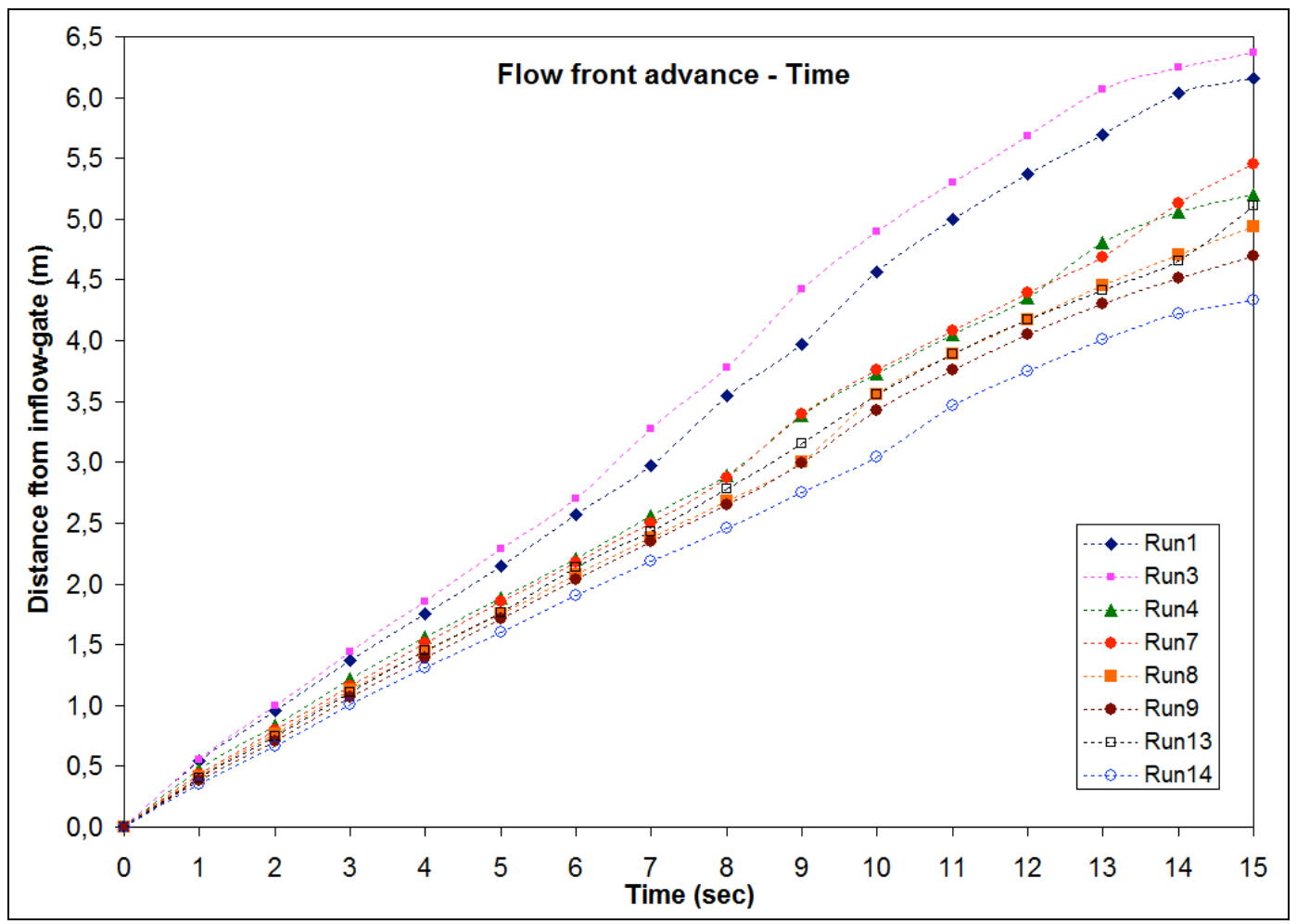

Fig. 21 


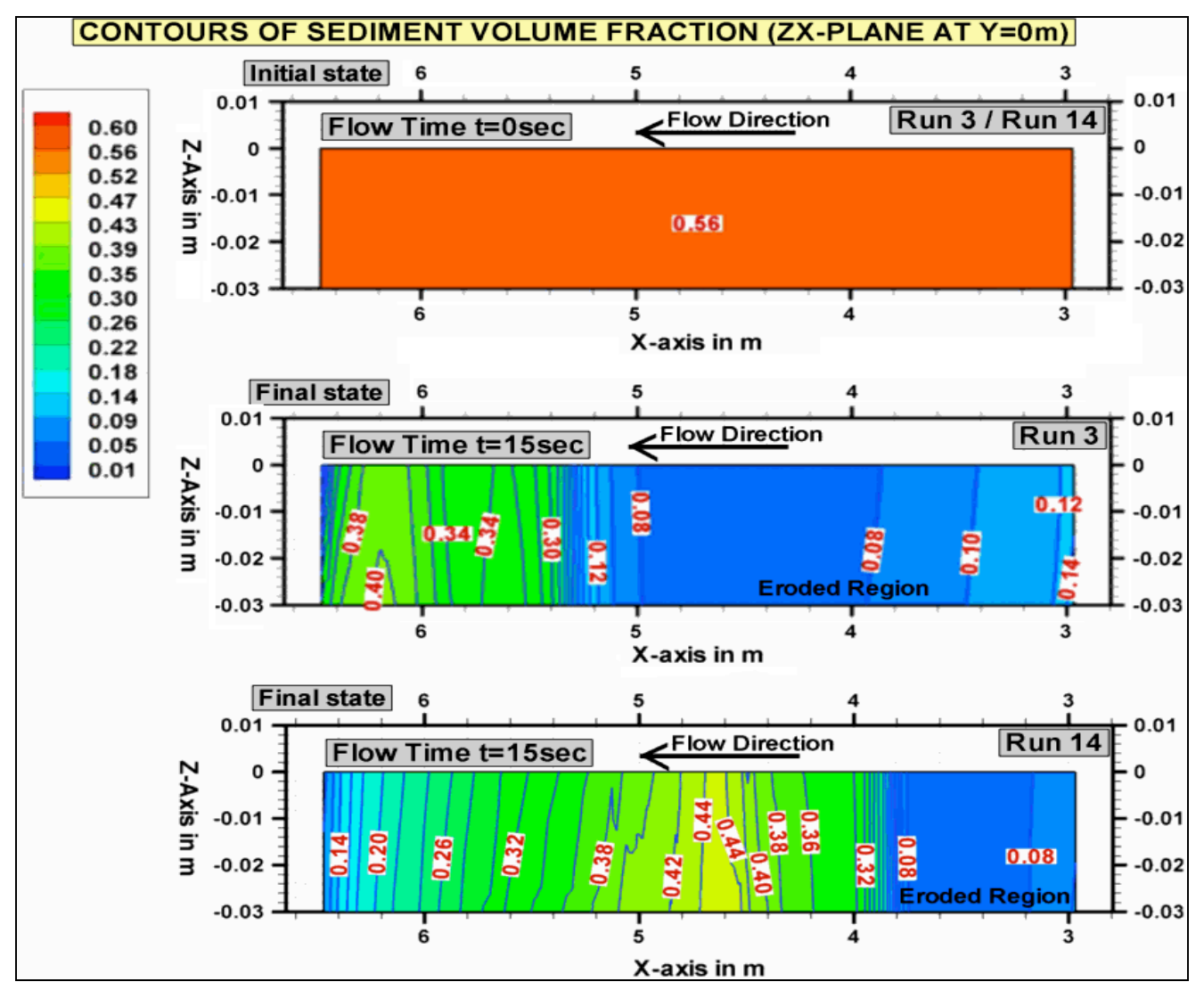

Fig. 22

Table 1

\begin{tabular}{|c|c|c|}
\hline Run & \% coarse $(\mathbf{6 9} \boldsymbol{\mu m})$ & \% fine $(\mathbf{2 5} \boldsymbol{\mu m})$ \\
\hline A & 0 & 100 \\
\hline B & 20 & 80 \\
\hline C & 40 & 60 \\
\hline D & 50 & 50 \\
\hline E & 60 & 40 \\
\hline F & 80 & 20 \\
\hline G & 100 & 0 \\
\hline
\end{tabular}


Table 2

\begin{tabular}{|c|c|c|c|c|c|c|c|}
\hline Run & $\begin{array}{l}\text { Mean } \\
\text { Grain } \\
\text { Size } \\
(\mu \mathrm{m})\end{array}$ & $\begin{array}{l}\text { Initial } \\
\text { Sediment } \\
\text { Volume } \\
\text { Fraction }\end{array}$ & $\begin{array}{c}\text { Water- } \\
\text { sediment } \\
\text { Mixture } \\
\text { Discharge } \\
\left(\mathrm{m}^{3} / \mathrm{sec}\right)\end{array}$ & $\begin{array}{c}\text { Max. } \\
\text { Channel } \\
\text { Slope } \\
\text { Angle } \\
\left(^{\circ}\right)\end{array}$ & $\begin{array}{l}\text { Slope } \\
\text { Type }\end{array}$ & $\begin{array}{l}\text { Ambient } \\
\text { Water } \\
\text { Temp. } \\
\left({ }^{\circ} \mathrm{C}\right)\end{array}$ & $\begin{array}{c}\text { Inflow } \\
\text { Velocity* } \\
\text { (m/sec) }\end{array}$ \\
\hline 1 & 235 & 0.27 & 0.0078 & 8.6 & Smooth & 15 & 1.24 \\
\hline 3 & 235 & 0.35 & 0.0078 & 8.6 & Smooth & 13 & 1.24 \\
\hline 4 & 235 & 0.14 & 0.0078 & 8.6 & Smooth & 14 & 1.24 \\
\hline 7 & 40 & 0.27 & 0.0078 & 3.7 & Smooth & $15 \dagger$ & 1.24 \\
\hline 8 & 40 & 0.21 & 0.0078 & 3.7 & Smooth & $15 \dagger$ & 1.24 \\
\hline 9 & 40 & 0.29 & 0.0052 & 3.7 & Rough & 13 & 0.82 \\
\hline 13 & 69 & 0.35 & 0.0052 & 3.7 & Rought: & 12 & 0.82 \\
\hline 14 & 69 & 0.21 & 0.0052 & 3.7 & Rough & 13 & 0.82 \\
\hline \multicolumn{8}{|c|}{$\begin{array}{l}\text { *Calculated by division of the Discharge value reported in the experin } \\
\text { gate Cross-sectional Area }\left(0.0063 \mathrm{~m}^{2}\right) \text {. } \\
\dagger \text { Assumed values (Not available in corresponding experimental runs). } \\
\ddagger \text { Roughness that corresponds to } 235 \mu \mathrm{m} \text { sand. }\end{array}$} \\
\hline
\end{tabular}

Table 3

\begin{tabular}{|c|c|c|c|c|c|}
\hline Run & $\begin{array}{c}\text { Initial } \\
\text { Sediment } \\
\text { Concentration } \\
\text { (Vol. \%) }\end{array}$ & $\begin{array}{l}\text { Experimental } \\
\text { Mean } \\
\text { Height } \\
\text { of T.C. } \\
(\mathbf{m})^{*}\end{array}$ & $\begin{array}{c}\text { Experimental } \\
\text { Dense } \\
\text { Layer } \\
\text { Thickness } \\
\text { (m)* }\end{array}$ & $\begin{array}{c}\text { Numerical } \\
\text { Mean } \\
\text { Height } \\
\text { of T.C. } \\
\text { (m)** }\end{array}$ & $\begin{array}{c}\text { Numerical } \\
\text { Dense } \\
\text { Layer } \\
\text { Thickness } \\
\text { (m) } * * *\end{array}$ \\
\hline 4 & 14 & 0.34 & & & 0.16 \\
\hline 1 & 27 & 0.34 & 0.12 & 0.29 & 0.16 \\
\hline 3 & 35 & 0.28 & 0.14 & 0.28 & 0.16 \\
\hline \multicolumn{6}{|c|}{$\begin{array}{l}* \text { Measured at the video window at the end of the quasi-steady period }(\sim 15 \mathrm{sec}) . \\
* * \text { Calculated at the middle of the video window position (for flow time } t=15 \mathrm{sec}), \text { using } \\
\text { Equation }(16) . \\
* * * \text { Graphically calculated at the middle of the video window position (for flow time } t=15 \\
\text { sec), as the distance from bed that the vertical concentration profile graph changes slope. }\end{array}$} \\
\hline
\end{tabular}

